\title{
Bee Venom Acupuncture Effects on Pain and Its Mechanisms: An Updated Review
}

\author{
Soo-Hyun Sung ${ }^{1(\mathbb{D})}$ and Gihyun Lee ${ }^{2, *(\mathbb{D}}$ \\ 1 Department of Policy Development, National Development Institute of Korean Medicine, Seoul 04554, Korea; \\ koyote10010@nikom.or.kr \\ 2 College of Korean Medicine, Dongshin University, Naju 58245, Korea \\ * Correspondence: glee@khu.ac.kr
}

Citation: Sung, S.-H.; Lee, G. Bee Venom Acupuncture Effects on Pain and Its Mechanisms: An Updated Review. Toxins 2021, 13, 608. https:// doi.org/10.3390/toxins13090608

Received: 31 July 2021

Accepted: 26 August 2021

Published: 29 August 2021

Publisher's Note: MDPI stays neutral with regard to jurisdictional claims in published maps and institutional affiliations.

Copyright: (c) 2021 by the authors. Licensee MDPI, Basel, Switzerland. This article is an open access article distributed under the terms and conditions of the Creative Commons Attribution (CC BY) license (https:// creativecommons.org/licenses/by/ $4.0 /)$.

\begin{abstract}
Bee venom (BV) is a complex natural toxin that contains various pharmaceutical compounds. Bee venom acupuncture (BVA), involving a BV injection into a certain acupuncture point, has been utilized to relieve a range of pain conditions. Regardless of whether pain is caused by disease or injury, if not effectively treated, pain can exert a detrimental effect on all aspects of life. In the past decade, many researchers have investigated the anti-nociceptive effects of BVA through clinical use and experimental evaluation. This report reviews the existing knowledge on the analgesic effects of BVA, focusing on musculoskeletal pain, inflammatory pain and neuropathic pain, and its analgesic mechanisms. Although further clinical trials are needed to clinical application of experimental results, this review will contribute to the standardization and generalization of BVA.
\end{abstract}

Keywords: bee venom; bee venom acupuncture; musculoskeletal pain; inflammatory pain; neuropathic pain; traditional; complementary and alternative medicine

Key Contribution: This article reviews recent evidence of the analgesic applications of BVA in treating musculoskeletal pain, inflammatory pain, and neuropathic pain.

\section{Introduction}

Pain is a worldwide health problem and is highly prevalent $[1,2]$. The prevalence of pain is estimated at $20 \%$ [3-5]. Depending on the cause, such as diseases or injury, pain intensity varies from mild to severe [6,7]. Regardless of the type, untreated pain can affect the quality of life and may lead to psychological, physical, and social consequences $[8,9]$.

Bee venom (BV) is an animal toxin that contains several active compounds, such as enzymes, peptides, non-peptide components, and biologically active amines. BV demonstrates the potential to be used as a beneficial pain treatment $[10,11]$. There are several types of BV therapies available, such as bee sting therapy, bee venom acupuncture (BVA), and the external use of BV products. Bee sting therapy involves the injection of BV through live bee stings on human skin. For this reason, this treatment modality also exhibits a high risk of developing adverse side effects [12]. On the other hand, BVA, which involves the administration of BV diluted with saline into a specific acupoint, has been used to treat different types of pain in the clinical fields of traditional, complementary, and alternative medicine [10] and does not exhibit the same adverse risks as those observed with bee sting therapy.

In previous studies, Chen et al. [13] reviewed papers published before 2010 regarding the pain reduction effects of $\mathrm{BV}$ injection, and its analgesic and anti-nociceptive mechanisms. They highlighted that therapeutic BV injection might be beneficial for certain patients but may also involve risks. In this review, we summarise information published over a decade of research conducted on the potential therapeutic applications and mechanisms of BVA for pain treatment. Thus far, painful conditions where therapeutic applications of BVA have been studied include musculoskeletal pain (e.g., shoulder pain and low back 
pain), inflammatory pain (e.g., osteoarthritis pain and arthritis pain), neuropathic pain (e.g., post-stroke pain, complex regional pain syndrome, refractory postherpetic neuralgia, mechanical allodynia, cold allodynia, and intervertebral disc disease-induced neurological pain), post-ischaemic pain, and prostatic pain. The summary is provided preceding the discussion section of our review, and in the order of clinical studies and experimental studies (Tables 1-6). 
Table 1. Clinical on therapeutic application of bee venom for musculoskeletal pain.

\begin{tabular}{|c|c|c|c|c|c|c|c|}
\hline Disease & $\begin{array}{l}\text { Study Design } \\
\text { (Number of } \\
\text { Patient or Animal } \\
\text { Treated by BV) }\end{array}$ & $\begin{array}{l}\text { Venom/ } \\
\text { Compound/ } \\
\text { (Bee Species) }\end{array}$ & $\begin{array}{c}\text { Intervention } \\
\text { (Acupoints, Dilution Ratio, Amount of Bee } \\
\text { Venom Use) }\end{array}$ & Main Results & $\begin{array}{l}\text { Mechanism/ } \\
\text { Molecular } \\
\text { Response }\end{array}$ & $\begin{array}{c}\text { Adverse Events of } \\
\text { BVA }\end{array}$ & Reference \\
\hline $\begin{array}{l}\text { Myofascial } \\
\text { shoulder pain }\end{array}$ & $\begin{array}{l}\text { Human, RCT } \\
\quad(n=50)\end{array}$ & BV (A. mellifera) & $\begin{array}{ll}\text { BVA } & \\
- & \text { acupoint: GB21 } \\
- & \text { concentration: not reported } \\
- & \text { 1 session: } 1 \mathrm{~mL} \\
- & \text { total } 2 \text { sessions: } 2 \mathrm{~mL} \\
\text { Needle free BVA } \\
-\quad \\
-\quad \text { acupoint: GB21 } \\
-\quad \text { concentration: not reported } \\
-\quad \text { session: } 1 \mathrm{~mL} \\
\quad \text { total } 2 \text { sessions: } 2 \mathrm{~mL}\end{array}$ & $\begin{array}{l}\text { BVA versus needle free BVA } \\
\text { - } \quad \text { significant effect of } \\
\text { VAS in both groups } \\
\text { ( } p \text { values were not } \\
\text { reported })\end{array}$ & $\begin{array}{l}\text { Not } \\
\text { reported }\end{array}$ & 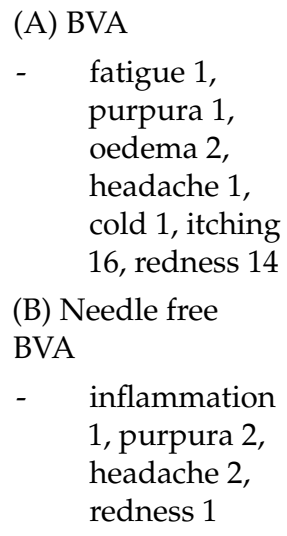 & [15] \\
\hline
\end{tabular}


Table 1. Cont.

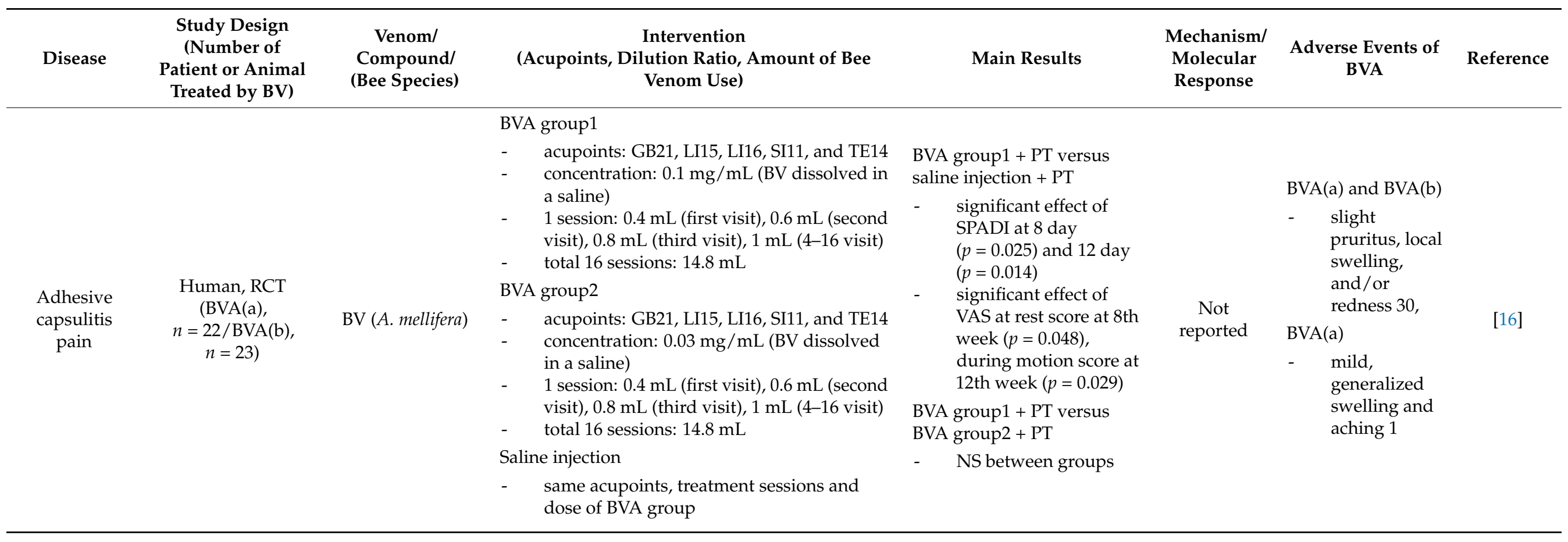


Table 1. Cont.

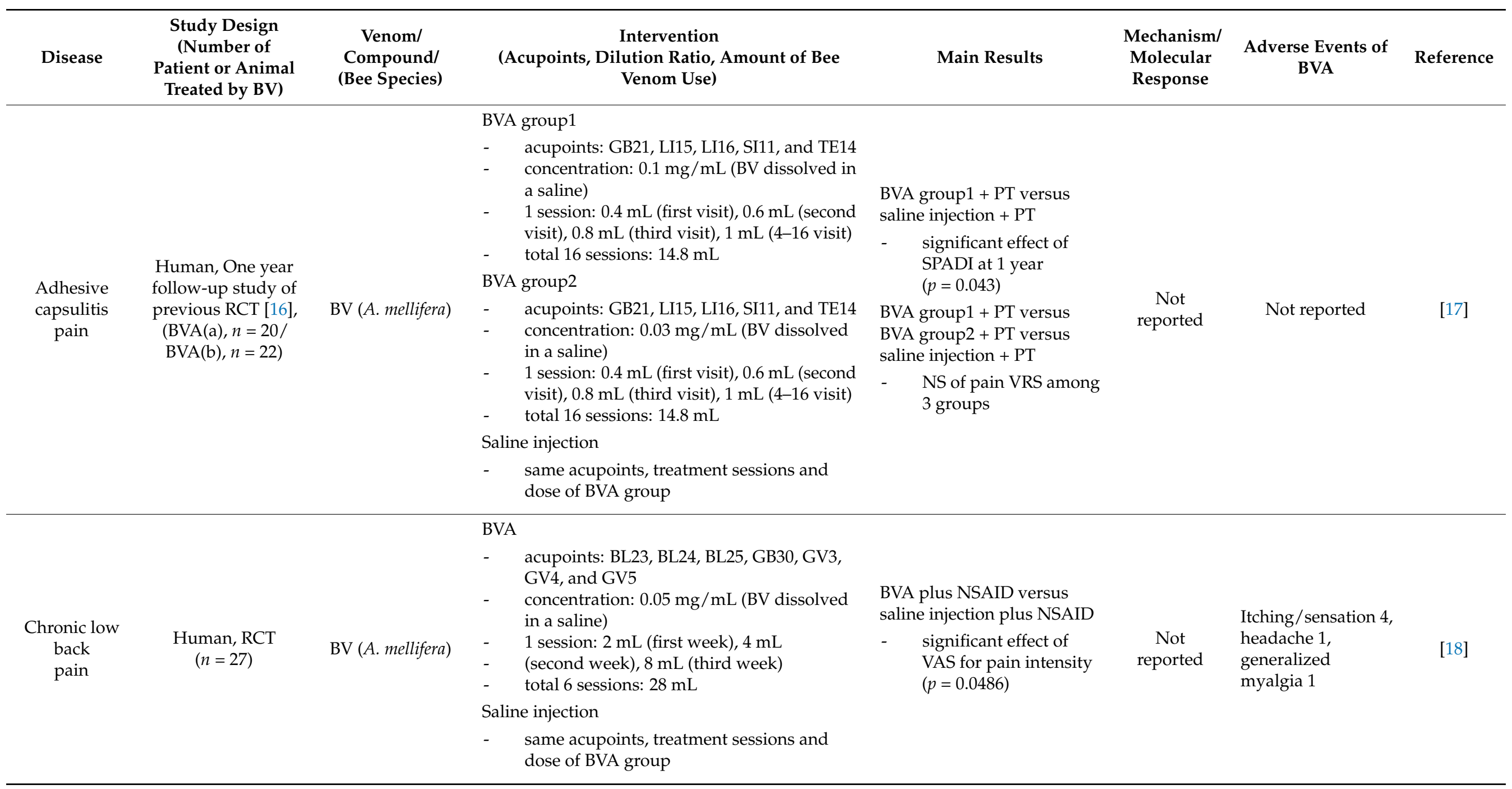


Table 1. Cont.

\begin{tabular}{|c|c|c|c|c|c|c|c|}
\hline Disease & $\begin{array}{l}\text { Study Design } \\
\text { (Number of } \\
\text { Patient or Animal } \\
\text { Treated by BV) }\end{array}$ & $\begin{array}{l}\text { Venom/ } \\
\text { Compound/ } \\
\text { (Bee Species) }\end{array}$ & $\begin{array}{c}\text { Intervention } \\
\text { (Acupoints, Dilution Ratio, Amount of Bee } \\
\text { Venom Use) }\end{array}$ & Main Results & $\begin{array}{l}\text { Mechanism/ } \\
\text { Molecular } \\
\text { Response }\end{array}$ & $\begin{array}{c}\text { Adverse Events of } \\
\text { BVA }\end{array}$ & Reference \\
\hline $\begin{array}{l}\text { Chronic low } \\
\text { back } \\
\text { SOFJApain }\end{array}$ & $\begin{array}{l}\text { Human, RCT } \\
\quad(n=60)\end{array}$ & $\mathrm{BV}$ (A. mellifera) & $\begin{array}{ll}\text { BVA } & \\
- & \text { acupoints: BL23, BL24, and BL25 } \\
- & \text { concentration: } 0.05 \mathrm{mg} / \mathrm{mL} \text { (BV dissolved } \\
- & \text { in a saline) } \\
- & \text { 1 session: } 0.6 \mathrm{~mL} \\
\text { Sotal } 8 \text { sessions: } 4.8 \mathrm{~mL} \\
\text { Saline injection } \\
-\quad \begin{array}{l}\text { same acupoints, treatment sessions and } \\
\text { dose of BVA group }\end{array}\end{array}$ & $\begin{array}{l}\text { BVA versus saline injection } \\
\text { - } \quad \text { significant effect of } \\
\text { VAS for pain intensity } \\
(p=0.0087)\end{array}$ & $\begin{array}{c}\text { Not } \\
\text { reported }\end{array}$ & $\begin{array}{l}\text { pruritus } 15 \text {, skin } \\
\text { flare } 5 \text {, } \\
\text { oedema } 4 \text {, skin } \\
\text { rash } 2\end{array}$ & [19] \\
\hline $\begin{array}{c}\text { Low back } \\
\text { pain }\end{array}$ & $\begin{array}{l}\text { Human, } \\
\text { Retrospective study } \\
\quad(n=524)\end{array}$ & BV (A. mellifera) & $\begin{array}{l}\text { BVA } \\
\text { - } \quad \text { acupoint: } 4-5 \text { acupoints around the lumbar } \\
\text { spine } \\
\text { - } \quad \text { concentration: } 0.1 \mathrm{mg} / \mathrm{mL} \text { (BV dissolved in } \\
\text { a saline) } \\
\text { - } \quad 1 \text { session: } 0.5-1 \mathrm{~mL} \\
\text { - } \quad \text { total treatment sessions: } 2.3 \pm 1.8\end{array}$ & $\begin{array}{l}\text { BVA }+ \text { herbal medicine }+ \\
\text { acupuncture }+ \text { chuna } \\
\text { - } \quad \text { average reduction in } \\
\quad \text { NRS was } 3.18-2.29 \\
\quad(95 \% \text { confidence } \\
\text { interval }[\mathrm{CI}], 2.99-3.38)\end{array}$ & $\begin{array}{c}\text { Not } \\
\text { reported }\end{array}$ & Allergic reactions 8 & [20] \\
\hline
\end{tabular}

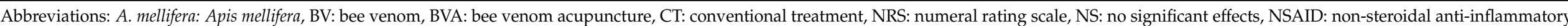
drugs, PT: physiotherapy, RCT: randomized controlled trials, SPADI: shoulder pain and disability index, VAS: visual analogue scale, VRS: verbal rating scale. 
Table 2. Clinical studies on therapeutic application of bee venom for inflammatory pain.

\begin{tabular}{|c|c|c|c|c|c|c|c|}
\hline Disease & $\begin{array}{c}\text { Study Design } \\
\text { (Number of } \\
\text { Patient or Animal } \\
\text { Treated by BV) }\end{array}$ & $\begin{array}{c}\text { Venom/ } \\
\text { Compound/ } \\
\text { (Bee Species) }\end{array}$ & $\begin{array}{c}\text { Intervention } \\
\text { (Dilution Ratio, Amount of Bee Venom Use) }\end{array}$ & Main Results & $\begin{array}{l}\text { Mechanism/ } \\
\text { Molecular } \\
\text { Response }\end{array}$ & $\begin{array}{l}\text { Adverse Events of } \\
\text { BVA }\end{array}$ & Reference \\
\hline $\begin{array}{c}\text { Knee } \\
\text { osteoarthritis } \\
\text { pain }\end{array}$ & $\begin{array}{l}\text { Human, RCT } \\
\quad(n=361)\end{array}$ & BV (A. mellifera) & 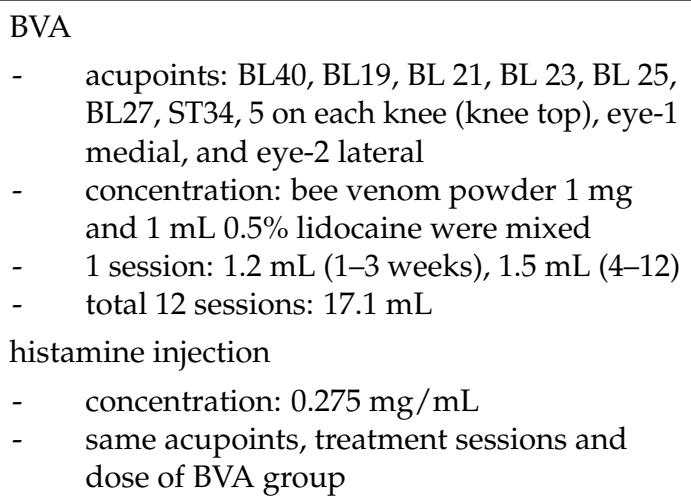 & $\begin{array}{l}\text { BVA versus histamine } \\
\text { injection } \\
\text { - } \quad \text { significant effect of } \\
\text { WOMAC pain score } \\
(p=0.0010)\end{array}$ & $\begin{array}{l}\text { Not } \\
\text { reported }\end{array}$ & $\begin{array}{l}\text { AEs } 177 \text {, injection } \\
\text { site AEs } 15\end{array}$ & [21] \\
\hline
\end{tabular}


Table 3. Experimental studies on therapeutic application of bee venom for inflammatory pain.

\begin{tabular}{|c|c|c|c|c|c|c|c|}
\hline Disease & $\begin{array}{l}\text { Study Design } \\
\text { (Number of } \\
\text { Patient or Animal } \\
\text { Treated by BV) }\end{array}$ & $\begin{array}{c}\text { Venom/ } \\
\text { Compound/ } \\
\text { (Bee Species) }\end{array}$ & $\begin{array}{c}\text { Intervention } \\
\text { (Dilution Ratio, Amount of Bee Venom Use) }\end{array}$ & Main Results & $\begin{array}{l}\text { Mechanism/ } \\
\text { Molecular Response }\end{array}$ & $\begin{array}{c}\text { Adverse } \\
\text { Events } \\
\text { of BVA }\end{array}$ & Reference \\
\hline
\end{tabular}


Table 3. Cont.

\begin{tabular}{|c|c|c|c|c|c|c|c|}
\hline Disease & $\begin{array}{c}\text { Study Design } \\
\text { (Number of } \\
\text { Patient or Animal } \\
\text { Treated by BV) }\end{array}$ & $\begin{array}{c}\text { Venom/ } \\
\text { Compound/ } \\
\text { (Bee Species) }\end{array}$ & $\begin{array}{c}\text { Intervention } \\
\text { (Dilution Ratio, Amount of Bee Venom Use) }\end{array}$ & Main Results & $\begin{array}{c}\text { Mechanism/ } \\
\text { Molecular Response }\end{array}$ & $\begin{array}{c}\text { Adverse } \\
\text { Events } \\
\text { of BVA }\end{array}$ & Reference \\
\hline $\begin{array}{l}\text { Arthritis } \\
\text { pain }\end{array}$ & $\begin{array}{c}\text { Male Wistar rats } \\
(150-160 \mathrm{~g}), \\
\text { collagen type II } \\
\text { induced arthritis } \\
\text { pain }(\mathrm{E} 1, n=5 / \mathrm{E} 2 \\
n=3)\end{array}$ & $\mathrm{BV}$ (A. mellifera) & 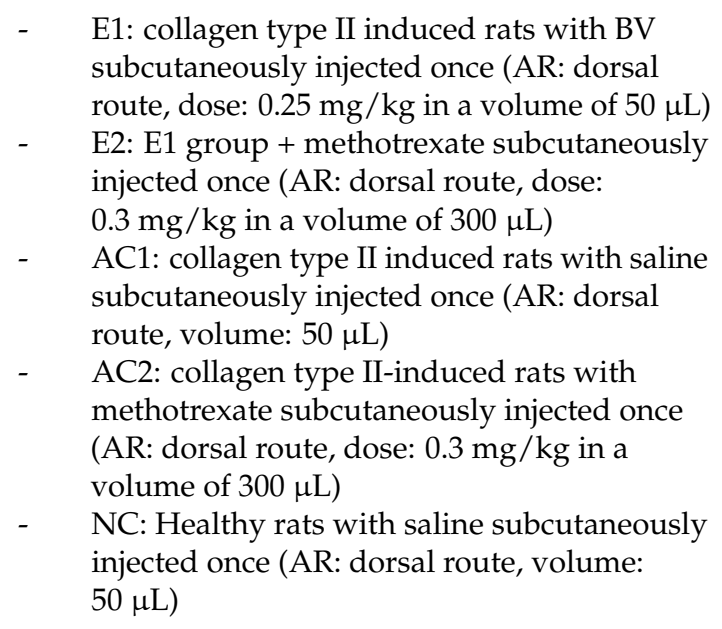 & $\begin{array}{l}\text { Arthritis pain (mechanical } \\
\text { threshold of hyperalgesia) } \\
\text { - } \quad \text { (AC1 vs. NC) decrease in } \\
\text { AC1 compared with NC } \\
\text { - } \quad \text { (E1 vs. NC) NS between E1 } \\
\text { and NC } \\
\text { - } \quad \text { (AC2 vs. NC) decrease in } \\
\text { AC2 compared with NC } \\
\text { (E2 vs. NC) decrease in E2 } \\
\text { compared with NC }\end{array}$ & $\begin{array}{l}\text { BV restores } \\
\text { APN in SF and } \\
\text { in soluble } \\
\text { fraction from } \\
\text { ST, and DPPIV } \\
\text { in solubilized } \\
\text { membrane- } \\
\text { bound fraction } \\
\text { from PBMCs }\end{array}$ & $\begin{array}{l}\text { Not re- } \\
\text { ported }\end{array}$ & [23] \\
\hline $\begin{array}{l}\text { Inflamm- } \\
\text { atory } \\
\text { pain }\end{array}$ & $\begin{array}{c}\text { Male ICR mice } \\
(20-25 \mathrm{~g}), \text { formalin } \\
\text { induced } \\
\text { inflammatory pain } \\
(\mathrm{E} 1, n=8 / \mathrm{E} 2, n=8)\end{array}$ & BV (A. mellifera) & $\begin{array}{l}\text { - } \quad \text { E1: formalin-induced mice with BV } \\
\text { subcutaneously injected once before formalin } \\
\text { injection (AR: ST36 acupoint, dose: } \\
0.8 \mathrm{mg} / \mathrm{kg}, \mathrm{BV} \text { dissolved in a volume of } \\
20 \mu \mathrm{L} \text { saline) } \\
\text { E2: formalin-induced mice with BV } \\
\text { subcutaneously injected once before formalin } \\
\text { injection (AR: ST36 acupoint, dose: } \\
0.08 \mathrm{mg} / \mathrm{kg}, \mathrm{BV} \text { dissolved in a volume of } \\
20 \mu \mathrm{L} \text { saline) } \\
\text { NC: formalin induced mice with saline } \\
\text { subcutaneously injected once before formalin } \\
\text { injection (AR: ST36 acupoint, volume: } 20 \mu \mathrm{L} \text { ) }\end{array}$ & $\begin{array}{l}\text { Inflammatory pain (paw licking } \\
\text { time) } \\
\text { - } \quad \text { (E1 vs. NC) NS (first } 10 \mathrm{~min} \text { ), } \\
\text { and } p<0.001 \text { (subsequent } \\
20 \mathrm{~min} \text { ) } \\
\text { - } \text { (E2 vs. NC) NS (first } 10 \mathrm{~min} \text { ), } \\
\text { and NS (subsequent } 20 \mathrm{~min} \text { ) }\end{array}$ & $\begin{array}{l}\text { BVA produces a } \\
\text { potent anti- } \\
\text { nociceptive } \\
\text { effect via the } \\
\text { activation of } \\
\text { spinal } \alpha-2 \\
\text { adrenoceptors }\end{array}$ & $\begin{array}{l}\text { Not re- } \\
\text { ported }\end{array}$ & [24] \\
\hline
\end{tabular}


Table 3. Cont.

\begin{tabular}{|c|c|c|c|c|c|c|c|}
\hline Disease & $\begin{array}{l}\text { Study Design } \\
\text { (Number of } \\
\text { Patient or Animal } \\
\text { Treated by BV) }\end{array}$ & $\begin{array}{c}\text { Venom/ } \\
\text { Compound/ } \\
\text { (Bee Species) }\end{array}$ & $\begin{array}{c}\text { Intervention } \\
\text { (Dilution Ratio, Amount of Bee Venom Use) }\end{array}$ & Main Results & $\begin{array}{l}\text { Mechanism/ } \\
\text { Molecular Response }\end{array}$ & $\begin{array}{l}\text { Adverse } \\
\text { Events } \\
\text { of BVA }\end{array}$ & Reference \\
\hline $\begin{array}{l}\text { Inflamm- } \\
\text { atory } \\
\text { pain }\end{array}$ & $\begin{array}{c}\text { Male ICR mice } \\
\text { (25-30 g), formalin } \\
\text { induced } \\
\text { inflammatory pain } \\
(\mathrm{E}, n=8 / \mathrm{AC} 1 \\
n=8 / \mathrm{AC} 2, n=8)\end{array}$ & BV (A. mellifera) & $\begin{array}{l}\text { E: formalin-induced mice with BV (AR: } \\
\text { ST36 acupoint, dose: } 0.8 \mathrm{mg} / \mathrm{kg}(1 \mathrm{~K}, \\
\text { diluted by saline with ratio of 1:1000) and } \\
\text { saline (AR: ST36 acupoint, volume: } 100 \mu \mathrm{L}) \\
\text { subcutaneously injected once after formalin } \\
\text { injection } \\
\text { AC1: formalin-induced mice with BV (AR: } \\
\text { ST36 acupoint, dose: } 0.8 \mathrm{mg} / \mathrm{kg}(1 \mathrm{~K}, \\
\text { diluted by saline with ratio of 1:1000) and } \\
\text { hydroxydopamine (AR: intraperitoneal } \\
\text { route, volume: } 100 \mu \mathrm{L}) \text { injected once after } \\
\text { formalin injection } \\
\text { AC2: formalin-induced mice with BV (AR: } \\
\text { ST36 acupoint, dose: } 0.8 \mathrm{mg} / \mathrm{kg}(1 \mathrm{~K}, \\
\text { diluted by saline with ratio of } 1: 1000) \text { and } \\
\text { epinephrine (AR: intraperitoneal route, } \\
\text { volume: } 100 \mu \mathrm{L}) \text { injected once after } \\
\text { formalin injection } \\
\text { NC: formalin- induced mice with saline } \\
\text { subcutaneously injected once after formalin } \\
\text { injection (AR: ST36 acupoint, volume: } \\
100 \mu \mathrm{L})\end{array}$ & 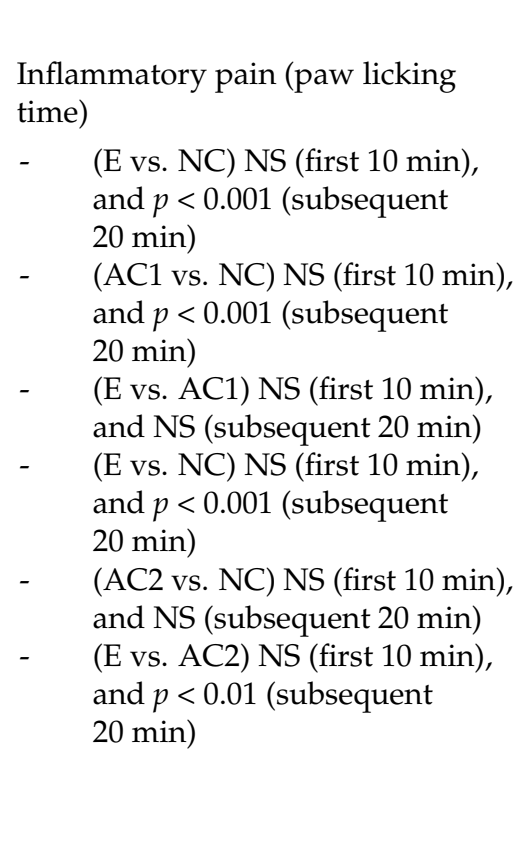 & $\begin{array}{l}\text { BVA performed } \\
\text { in combination } \\
\text { with } \\
\text { administration } \\
\text { of peripheral } \\
\beta \text {-adrenoceptor } \\
\text { antagonists } \\
\text { would be a } \\
\text { potential novel } \\
\text { strategy for the } \\
\text { pain } \\
\text { management }\end{array}$ & $\begin{array}{l}\text { Not re- } \\
\text { ported }\end{array}$ & [25] \\
\hline
\end{tabular}


Table 3. Cont.

\begin{tabular}{|c|c|c|c|c|c|c|c|c|}
\hline Disease & $\begin{array}{c}\text { Study Design } \\
\text { (Number of } \\
\text { Patient or Animal } \\
\text { Treated by BV) }\end{array}$ & $\begin{array}{c}\text { Venom/ } \\
\text { Compound/ } \\
\text { (Bee Species) }\end{array}$ & $\begin{array}{c}\text { Intervention } \\
\text { (Dilution Ratio, Amount of Bee Venom Use) }\end{array}$ & Main Results & \multicolumn{2}{|c|}{$\begin{array}{l}\text { Mechanism/ } \\
\text { Molecular Response }\end{array}$} & $\begin{array}{l}\text { Adverse } \\
\text { Events } \\
\text { of BVA }\end{array}$ & Reference \\
\hline \multicolumn{9}{|c|}{$\begin{array}{l}\text { Abbreviations: A. dorsata: Apis dorsata, A. mellifera: Apis mellifera, AC: active control group, APN: altered activity of basic, AR: administration route, BV: bee venom, BVA: bee venom acupuncture, DPPIV: } \\
\text { dipeptidyl peptidase IV, E: experimental group, FCA: Freund's complete adjuvant, IL-6: interleukin-6, NC: normal control group, NS: no significant effects, PBMCs: peripheral blood mononuclear cells, SF: } \\
\text { synovial fluid, ST: synovial tissue, TNF- } \alpha \text { : tumour necrosis factor- } \alpha \text {. }\end{array}$} \\
\hline Disease & $\begin{array}{l}\text { Study Design } \\
\text { (Number of } \\
\text { Patient or Animal } \\
\text { Treated by BV) }\end{array}$ & $\begin{array}{l}\text { Venom/ } \\
\text { Compound/ } \\
\text { (Bee Species) }\end{array}$ & $\begin{array}{c}\text { Intervention } \\
\text { (Dilution Ratio, Amount of Bee Venom Use) }\end{array}$ & Main Results & $\begin{array}{l}\text { Mechanism/ } \\
\text { Molecular } \\
\text { Response }\end{array}$ & $\begin{array}{r}\text { Adverse } \\
\text { B }\end{array}$ & $\begin{array}{l}\text { Events of } \\
\text { JA }\end{array}$ & Reference \\
\hline $\begin{array}{l}\text { Post-stroke } \\
\text { shoulder pain }\end{array}$ & $\begin{array}{l}\text { Human, systematic } \\
\text { review }(n=75)\end{array}$ & BV (A. mellifera) & 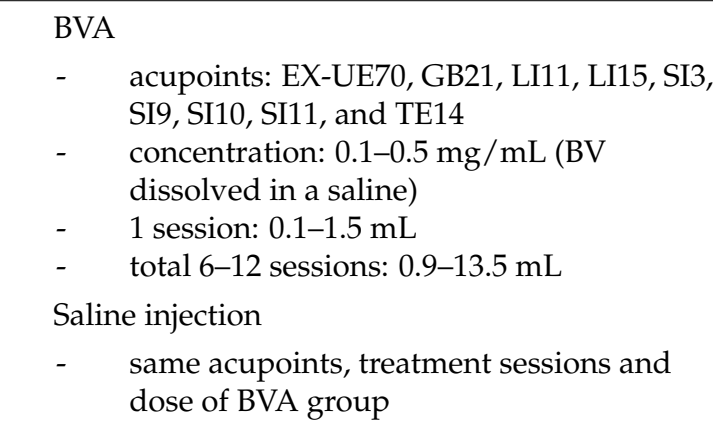 & $\begin{array}{l}\text { BVA versus saline injection } \\
\text { - } \quad \text { significant effect of } \\
\text { VAS }(p=0.02)\end{array}$ & $\begin{array}{l}\text { Not } \\
\text { reported }\end{array}$ & $\begin{array}{r}\text { Pain 2, } \\
\text { burning }\end{array}$ & $\begin{array}{l}\text { ruritus } 8 \\
\text { ensation } 3\end{array}$ & [27] \\
\hline
\end{tabular}


Table 4. Cont.

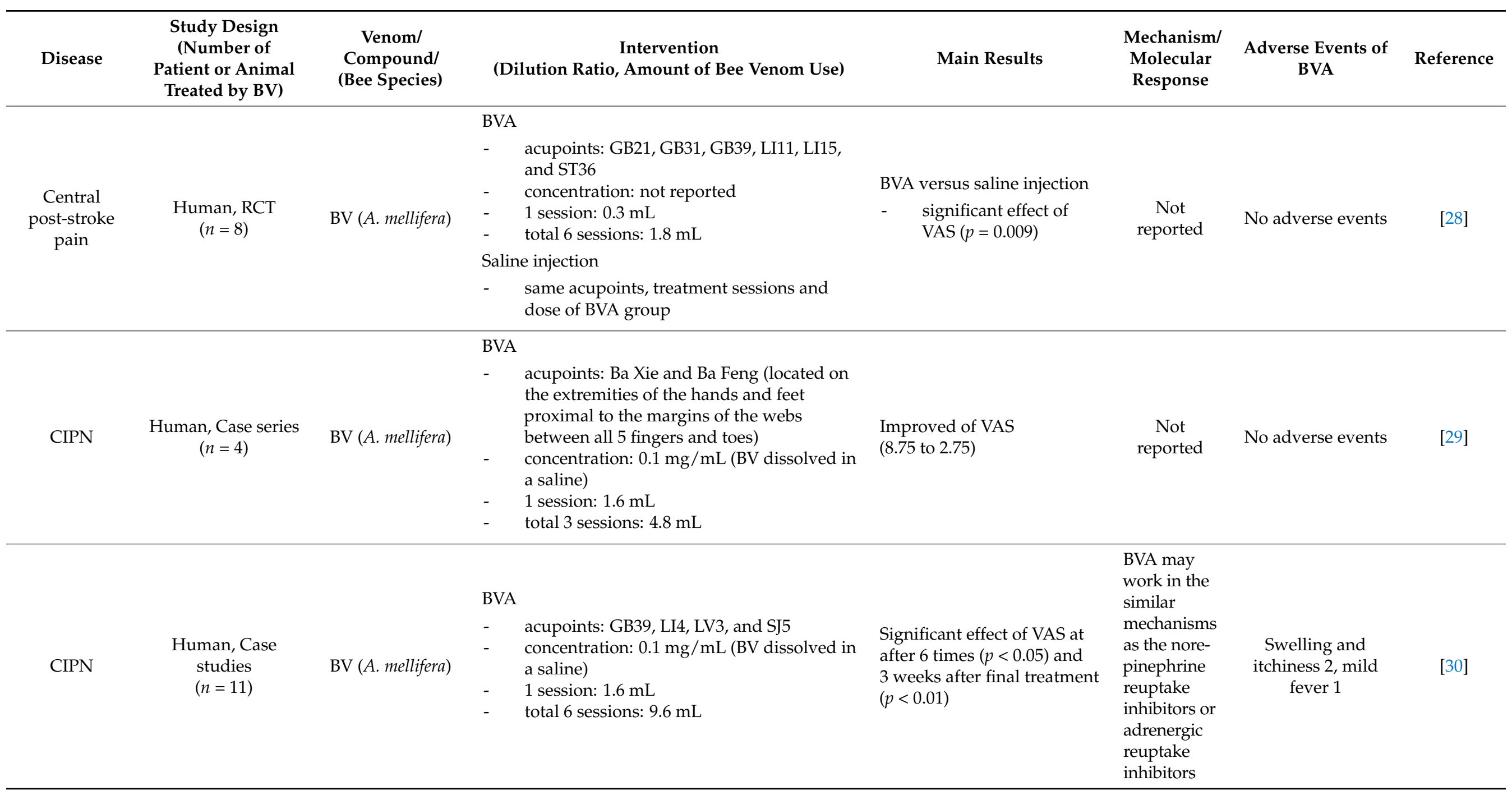


Table 4. Cont.

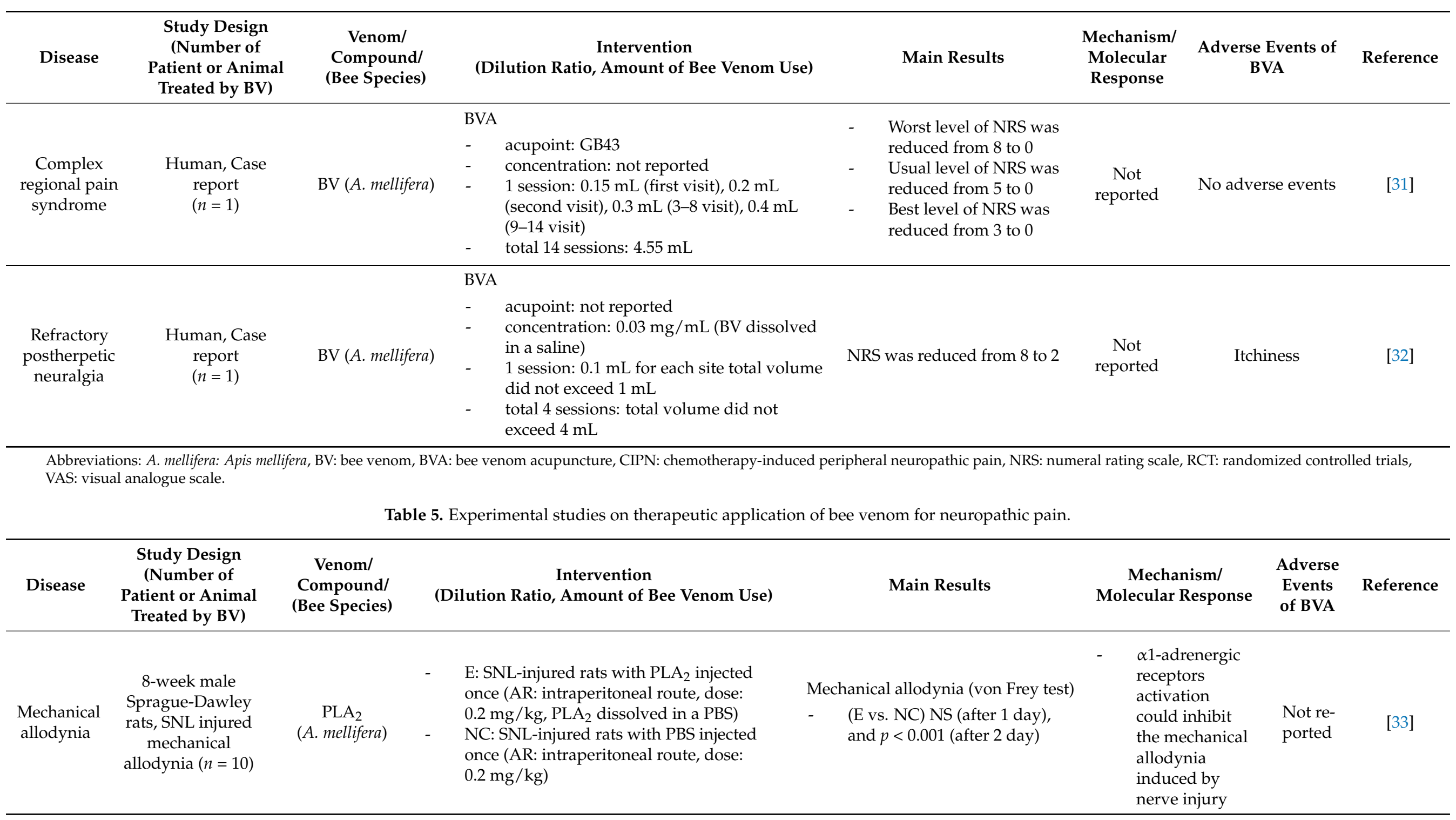


Table 5. Cont.

\begin{tabular}{|c|c|c|c|c|c|c|c|}
\hline Disease & $\begin{array}{c}\text { Study Design } \\
\text { (Number of } \\
\text { Patient or Animal } \\
\text { Treated by BV) }\end{array}$ & $\begin{array}{c}\text { Venom/ } \\
\text { Compound/ } \\
\text { (Bee Species) }\end{array}$ & $\begin{array}{c}\text { Intervention } \\
\text { (Dilution Ratio, Amount of Bee Venom Use) }\end{array}$ & Main Results & $\begin{array}{l}\text { Mechanism/ } \\
\text { Molecular Response }\end{array}$ & $\begin{array}{c}\text { Adverse } \\
\text { Events } \\
\text { of BVA }\end{array}$ & Reference \\
\hline $\begin{array}{l}\text { Mechanical } \\
\text { and cold } \\
\text { allodynia }\end{array}$ & $\begin{array}{l}\text { Male Sprague } \\
\text { Dawley rats } \\
(180-200 \mathrm{~g}), \mathrm{SNL} \\
\text { injured mechanical } \\
\text { and cold allodynia } \\
(\mathrm{E} 1, n=7 / \mathrm{E} 2, n=7)\end{array}$ & BV (A. mellifera $)$ & $\begin{array}{l}\text { E1: SNL-injured rats with BV injected once } \\
\text { (AR: peri-neural sheath of the L5 and L6 } \\
\text { spinal nerves, dose: } 0.1 \mathrm{mg} / \mathrm{kg}, \mathrm{BV} \\
\text { dissolved in } 0.9 \% \text { saline at concentrations } \\
\text { of } 2 \mu \mathrm{g} / \mu \mathrm{L}) \\
\text { E2: } \\
\text { (ANL-injured rats with BV injected once } \\
\text { spinal nerves, dose: } 0.05 \mathrm{mg} / \mathrm{kg}, \mathrm{BV} \\
\text { dissolved in } 0.9 \% \text { saline at concentrations } \\
\text { of } 1 \mu \mathrm{g} / \mu \mathrm{L}) \\
\text { NC: } \mathrm{SNL}-\text { injured rats with saline injected } \\
\text { once (AR: peri-neural sheath of the L5 and } \\
\text { L6 spinal nerves, dose: not reported) }\end{array}$ & 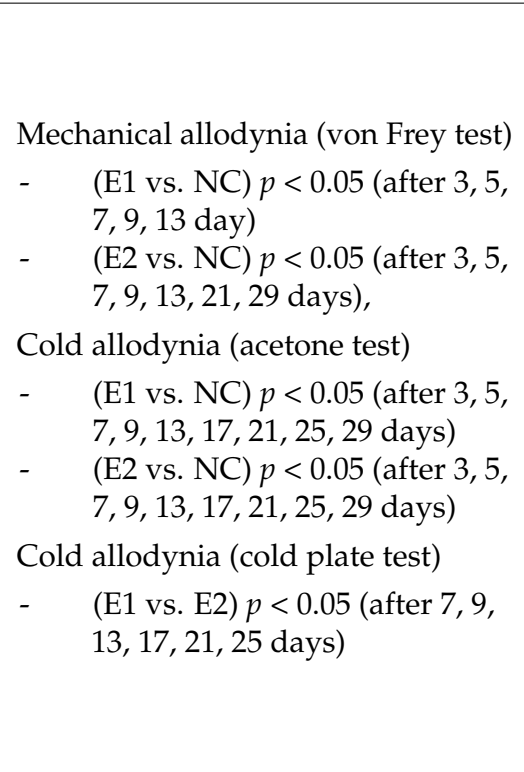 & $\begin{array}{l}\text { (mechanical } \\
\text { allodynia) } \\
\text { - } \quad \text { not reported. } \\
\text { (cold allodynia) } \\
\text { - } \quad \text { The expression } \\
\text { of TRPM8 } \\
\text { receptors was } \\
\text { significantly } \\
\text { reduced after } \\
\text { BVA } \\
\text { pretreatment, } \\
\text { indicating that } \\
\text { both TRPA1 } \\
\text { and TRPM8 are } \\
\text { involved in the } \\
\text { development of } \\
\text { cold allodynia } \\
\text { in the SNL } \\
\text { injury model }\end{array}$ & $\begin{array}{l}\text { Not re- } \\
\text { ported }\end{array}$ & [34] \\
\hline $\begin{array}{l}\text { Mechanical } \\
\text { allodynia }\end{array}$ & $\begin{array}{l}\text { 6-week male } \\
\text { Sprague-Dawley } \\
\text { rats }(180-210 \mathrm{~g}), \\
\text { paclitaxel induced } \\
\text { mechanical } \\
\text { allodynia }(n=14)\end{array}$ & BV (A. mellifera) & $\begin{array}{l}\text { - E: paclitaxel-induced rats with BV injected } \\
\text { once (AR: ST36 acupoint, dose: } 1.0 \mathrm{mg} / \mathrm{kg} \text {, } \\
\text { BV dissolved in a PBS) } \\
\text { NC: paclitaxel-induced rats with PBS } \\
\text { injected once (AR: ST36 acupoint, dose: not } \\
\text { reported) }\end{array}$ & $\begin{array}{l}\text { Mechanical allodynia (von Frey test) } \\
\text { (E1 vs. NC) } p<0.05 \text { (after } 1 \mathrm{~h} \text { ), } \\
p<0.01 \text { (after } 2 \mathrm{~h} \text { ), NS (after } 4 \\
\text { h), and NS (after } 6 \text { days) }\end{array}$ & $\begin{array}{l}\text { - } \begin{array}{l}\text { The action of } \\
\text { spinal } \\
\alpha 2 \text {-adrenergic } \\
\text { receptor, but } \\
\text { not }\end{array} \\
\alpha 1 \text {-adrenergic } \\
\text { receptor, is involved } \\
\text { in the mechanism of } \\
\text { analgesic effect. }\end{array}$ & $\begin{array}{l}\text { Not re- } \\
\text { ported }\end{array}$ & {$[35]$} \\
\hline
\end{tabular}


Table 5. Cont.

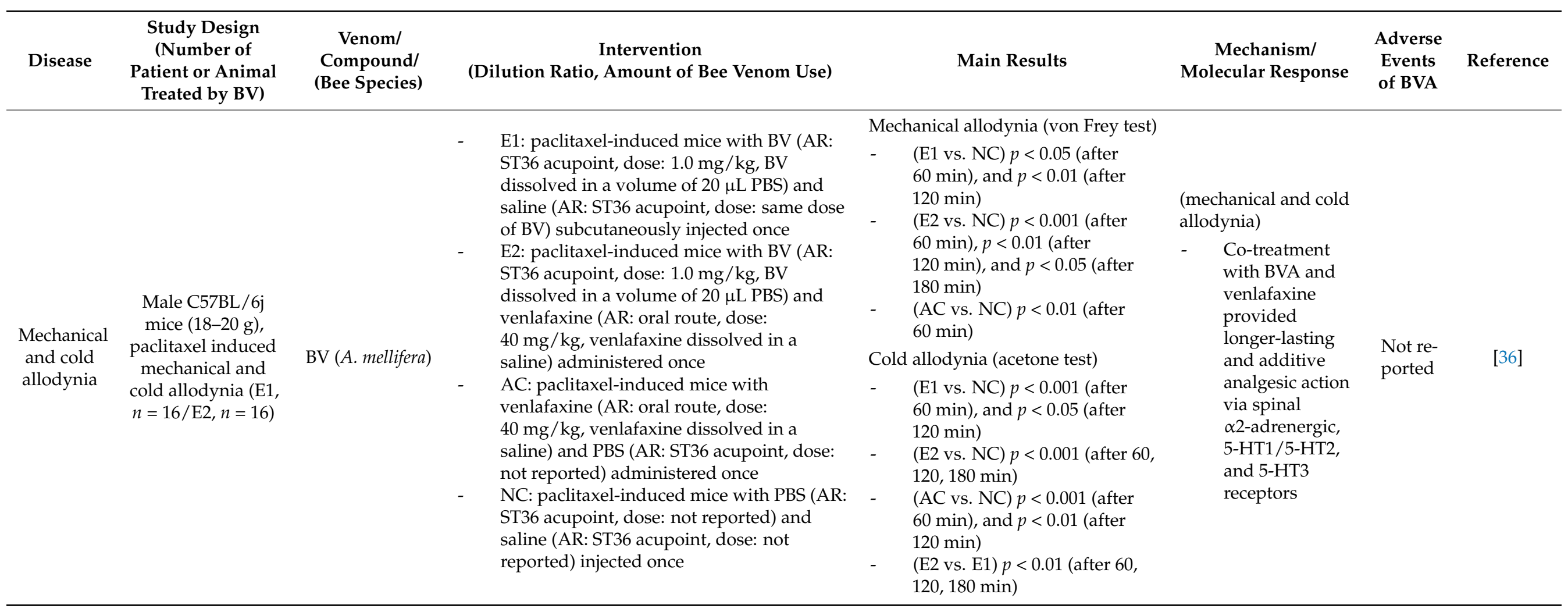


Table 5. Cont.

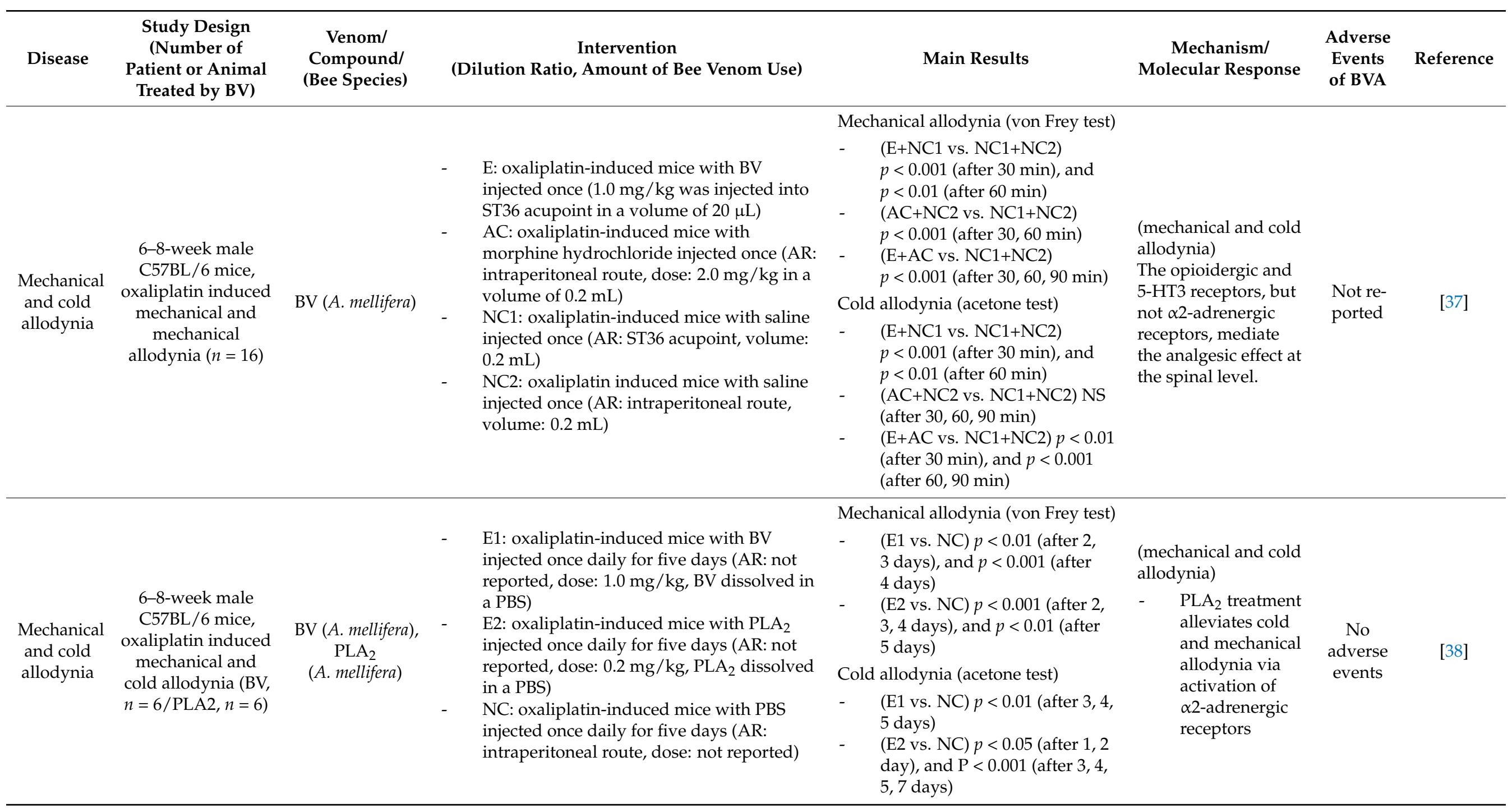


Table 5. Cont.

\begin{tabular}{|c|c|c|c|c|c|c|c|}
\hline Disease & $\begin{array}{l}\text { Study Design } \\
\text { (Number of } \\
\text { Patient or Animal } \\
\text { Treated by BV) }\end{array}$ & $\begin{array}{c}\text { Venom/ } \\
\text { Compound/ } \\
\text { (Bee Species) }\end{array}$ & $\begin{array}{c}\text { Intervention } \\
\text { (Dilution Ratio, Amount of Bee Venom Use) }\end{array}$ & Main Results & $\begin{array}{l}\text { Mechanism/ } \\
\text { Molecular Response }\end{array}$ & $\begin{array}{l}\text { Adverse } \\
\text { Events } \\
\text { of BVA }\end{array}$ & Reference \\
\hline $\begin{array}{l}\text { Mechanical } \\
\text { and cold } \\
\text { allodynia }\end{array}$ & $\begin{array}{l}\text { 6-8-week male } \\
\text { C57BL/6 mice, } \\
\text { oxaliplatin induced } \\
\text { mechanical and } \\
\text { cold allodynia } \\
\quad(n=13)\end{array}$ & $\begin{array}{c}\mathrm{PLA}_{2} \\
\text { (A. mellifera) }\end{array}$ & $\begin{array}{l}\text { E: oxaliplatin-induced mice with PLA } \\
\text { injected once daily for five days before } \\
\text { oxaliplatin was administered (AR: } \\
\text { intraperitoneal route, dose: } 0.2 \mathrm{mg} / \mathrm{kg} \text { ) } \\
\text { NC1: oxaliplatin-induced mice with PBS } \\
\text { injected once daily for five days before } \\
\text { oxaliplatin was administered (AR: } \\
\text { intraperitoneal route, dose: same dose of E } \\
\text { group) } \\
\text { NC2: mice injected with PBS (AR: } \\
\text { intraperitoneal route, dose: same dose of E } \\
\text { group) once daily for five days before } 5 \% \\
\text { glucose (AR: intraperitoneal route, volume: } \\
\text { not exceed } 0.5 \mathrm{~mL} \text { ) was administered }\end{array}$ & $\begin{array}{l}\text { Mechanical allodynia (von Frey test) } \\
-\quad \text { (E vs. NC1) } p<0.001 \text { (after } \\
3 \text { days) } \\
\text { - } \quad \text { E vs. NC2) NS (after 3, 5, } \\
7 \text { days) } \\
\text { Cold allodynia (acetone test) } \\
-\quad \text { (E vs. NC1) } p<0.001 \text { (after 3, } \\
\text { 5 days) } \\
\text { (E vs. NC2) NS (after 3, 5, } \\
7 \text { days) }\end{array}$ & $\begin{array}{l}\text { (mechanical and cold } \\
\text { allodynia) } \\
\text { - } \quad \text { PLA }_{2} \text { may } \\
\text { prevent } \\
\text { oxaliplatin- } \\
\text { induced } \\
\text { neuropathic } \\
\text { pain by } \\
\text { suppressing } \\
\text { immune } \\
\text { responses in the } \\
\text { DRG by Tregs }\end{array}$ & $\begin{array}{l}\text { Not re- } \\
\text { ported }\end{array}$ & [39] \\
\hline
\end{tabular}


Table 5. Cont.

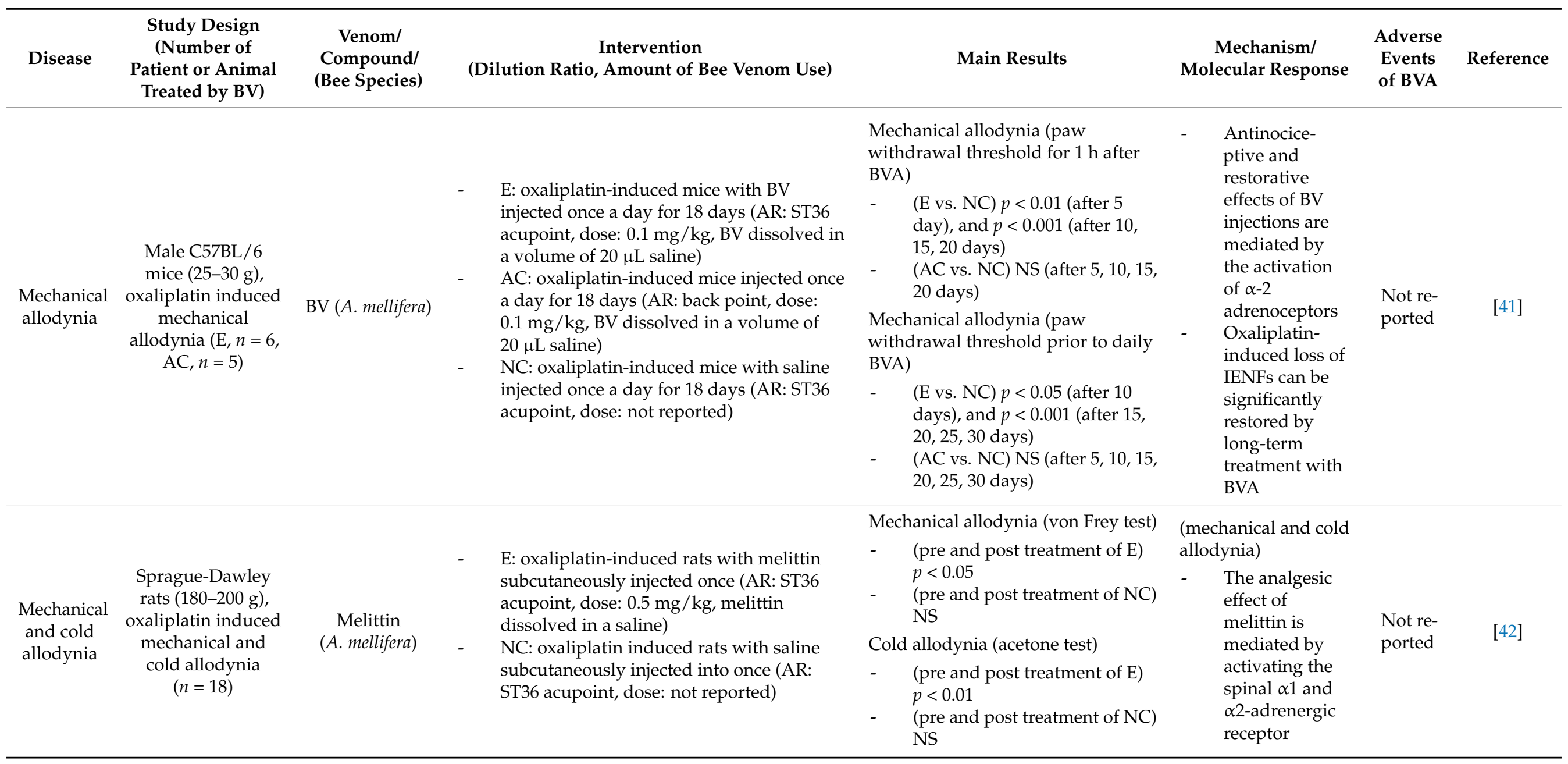


Table 5. Cont.

\begin{tabular}{|c|c|c|c|c|c|c|c|}
\hline Disease & $\begin{array}{c}\text { Study Design } \\
\text { (Number of } \\
\text { Patient or Animal } \\
\text { Treated by BV) }\end{array}$ & $\begin{array}{c}\text { Venom/ } \\
\text { Compound/ } \\
\text { (Bee Species) }\end{array}$ & $\begin{array}{c}\text { Intervention } \\
\text { (Dilution Ratio, Amount of Bee Venom Use) }\end{array}$ & Main Results & $\begin{array}{l}\text { Mechanism/ } \\
\text { Molecular Response }\end{array}$ & $\begin{array}{c}\text { Adverse } \\
\text { Events } \\
\text { of BVA }\end{array}$ & Reference \\
\hline $\begin{array}{l}\text { Mechanical } \\
\text { and cold } \\
\text { allodynia }\end{array}$ & $\begin{array}{c}\text { Male } \\
\text { Sprague-Dawley } \\
\text { rats, oxaliplatin } \\
\text { induced } \\
\text { mechanical and } \\
\text { cold allodynia (E1, } \\
n=10 / \mathrm{E} 2, n=10)\end{array}$ & BV (A. mellifera) & $\begin{array}{l}\text { - } \quad \text { E1: oxaliplatin-induced rats with BV } \\
\text { subcutaneously injected once (AR: ST36 } \\
\text { acupoint, dose: } 0.1 \mathrm{mg} / \mathrm{kg}, \mathrm{BV} \text { dissolved in } \\
\text { PBS at concentrations of } 1 \mathrm{mg} / \mathrm{mL}) \\
\text { E2: oxaliplatin-induced rats with BV } \\
\text { subcutaneously injected once (AR: ST36 } \\
\text { acupoint, dose: } 1.0 \mathrm{mg} / \mathrm{kg}, \mathrm{BV} \text { dissolved in } \\
\text { PBS at concentrations of } 1 \mathrm{mg} / \mathrm{mL}) \\
\text { NC: oxaliplatin-induced rats with PBS } \\
\text { subcutaneously injected once (AR: ST36 } \\
\text { acupoint, dose: not reported) }\end{array}$ & 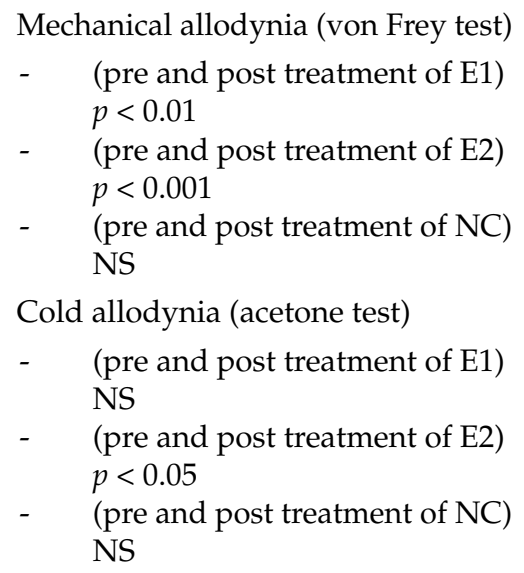 & $\begin{array}{l}\text { (mechanical and cold } \\
\text { allodynia) } \\
\text { - } \quad \text { BVA may } \\
\text { attenuate } \\
\text { oxaliplatin- } \\
\text { induced } \\
\text { neuropathic } \\
\text { pain by altering } \\
\text { the action } \\
\text { potential } \\
\text { threshold in } \\
\text { A-fibre dorsal } \\
\text { root ganglia } \\
\text { neurons. }\end{array}$ & $\begin{array}{l}\text { Not re- } \\
\text { ported }\end{array}$ & [43] \\
\hline
\end{tabular}


Table 5. Cont.

\begin{tabular}{|c|c|c|c|c|c|c|c|}
\hline Disease & $\begin{array}{l}\text { Study Design } \\
\text { (Number of } \\
\text { Patient or Animal } \\
\text { Treated by BV) }\end{array}$ & $\begin{array}{c}\text { Venom/ } \\
\text { Compound/ } \\
\text { (Bee Species) }\end{array}$ & $\begin{array}{c}\text { Intervention } \\
\text { (Dilution Ratio, Amount of Bee Venom Use) }\end{array}$ & Main Results & $\begin{array}{l}\text { Mechanism/ } \\
\text { Molecular Response }\end{array}$ & $\begin{array}{c}\text { Adverse } \\
\text { Events } \\
\text { of BVA }\end{array}$ & Reference \\
\hline $\begin{array}{l}\text { Mechanical } \\
\text { allodynia }\end{array}$ & $\begin{array}{l}\text { Male Sprague } \\
\text { Dawley rats } \\
(180-200 \mathrm{~g}), \\
\text { laminectomy was } \\
\text { performed between } \\
\text { the T11 to T12 } \\
\text { vertebral segments } \\
\quad(n=8)\end{array}$ & BV (A. mellifera) & $\begin{array}{l}\text { E: spinal cord injury rats with BV injected } \\
\text { subcutaneously twice a day from } 15 \text { to } \\
20 \text { days post-surgery (AR: ST36 acupoint, } \\
\text { dose: } 0.25 \mathrm{mg} / \mathrm{kg} \text {, BV dissolved in a } \\
\text { volume of } 50 \mu \mathrm{L} \text { saline) } \\
\text { NC: spinal cord injury rats with saline } \\
\text { injected subcutaneously twice a day from } \\
15 \text { to } 20 \text { days post-surgery (AR: ST36 } \\
\text { acupoint, dose: not reported) }\end{array}$ & $\begin{array}{l}\text { Mechanical allodynia (ipsilateral } \\
\text { paw) } \\
\text { (E vs. NC) NS (after } 1 \text { day), } \\
p<0.05 \text { (after } 3 \text { days), } p<0.05 \\
\text { (after } 5 \text { days), NS (after } 7 \\
\text { days), NS (after } 10 \text { day), and } \\
\text { NS (after } 14 \text { day) }\end{array}$ & $\begin{array}{l}\text { Reduction in } \\
\text { mechanical } \\
\text { allodynia is } \\
\text { mediated by } \\
\text { the suppression } \\
\text { of spinal } \\
\text { astrocyte or } \\
\text { microglia } \\
\text { activation }\end{array}$ & $\begin{array}{l}\text { Not re- } \\
\text { ported }\end{array}$ & [45] \\
\hline $\begin{array}{l}\text { Mechanical } \\
\text { and cold } \\
\text { allodynia }\end{array}$ & $\begin{array}{l}\text { Male } \\
\text { Sprague-Dawley } \\
\text { rats (190-210 g), } \\
\text { CIPN model was } \\
\text { established using } \\
\text { daily vincristine } \\
\text { infusions (E1, } \\
n=12 / \mathrm{E} 2, \\
n=12 / \mathrm{E} 3, n=12)\end{array}$ & $\begin{array}{c}\text { BV (A. mellifera }) \\
\text { PLA }_{2} \\
(\text { A. mellifera }) \\
\text { melittin } \\
(\text { A. mellifera })\end{array}$ & $\begin{array}{l}\text { - } \quad \text { E1: vincristine-induced rats with BV } \\
\text { subcutaneously injected once (AR: ST36 } \\
\text { acupoint, dose: } 1.0 \mathrm{mg} / \mathrm{kg} \text {, BV dissolved in } \\
\text { a volume of } 50 \mu \mathrm{L} \text { saline) } \\
\text { E2: vincristine-induced rats with melittin } \\
\text { subcutaneously injected once (AR: ST36 } \\
\text { acupoint, dose: } 0.5 \mathrm{mg} / \mathrm{kg} \text {, melittin } \\
\text { dissolved in a volume of } 50 \mu \mathrm{L} \text { saline) } \\
\text { E3: vincristine-induced rats with PLA } 2 \\
\text { (0.12 } \mathrm{mg} / \mathrm{kg} \text { ) subcutaneously injected once } \\
\text { (AR: ST36 acupoint, dose: } 0.12 \mathrm{mg} / \mathrm{kg} \text {, } \\
\text { PLA } \mathrm{A}_{2} \text { dissolved in a volume of } 50 \mu \mathrm{L} \text { saline) } \\
\text { NC: vincristine-induced rats with mice } \\
\text { with saline subcutaneously injected once } \\
\text { (AR: ST36 acupoint, dose: not reported) }\end{array}$ & 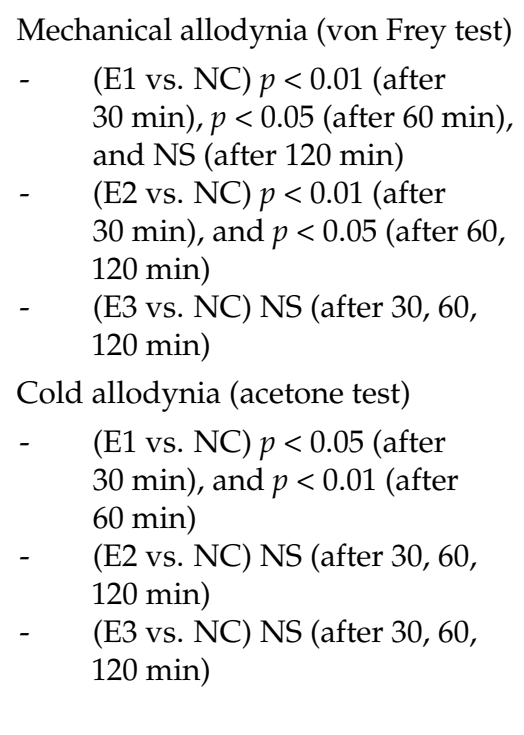 & $\begin{array}{l}\text { (mechanical and cold } \\
\text { allodynia) } \\
\text { - } \quad \text { BVA } \\
\text { manoeuvre } \\
\text { attenuated the } \\
\text { hyperexcitation } \\
\text { of spinal WDR } \\
\text { neurons in rats } \\
\text { with } \\
\text { neuropathy } \\
\text { BVA-induced } \\
\text { analgesia was } \\
\text { mediated by } \\
\text { the descending } \\
\text { noradrenergic } \\
\text { pathway, which } \\
\text { mainly } \\
\text { originates from } \\
\text { the LC }\end{array}$ & $\begin{array}{l}\text { Not re- } \\
\text { ported }\end{array}$ & [46] \\
\hline
\end{tabular}


Table 5. Cont.

\begin{tabular}{|c|c|c|c|c|c|c|c|}
\hline Disease & $\begin{array}{l}\text { Study Design } \\
\text { (Number of } \\
\text { Patient or Animal } \\
\text { Treated by BV) }\end{array}$ & $\begin{array}{c}\text { Venom/ } \\
\text { Compound/ } \\
\text { (Bee Species) }\end{array}$ & $\begin{array}{c}\text { Intervention } \\
\text { (Dilution Ratio, Amount of Bee Venom Use) }\end{array}$ & Main Results & $\begin{array}{l}\text { Mechanism/ } \\
\text { Molecular Response }\end{array}$ & $\begin{array}{l}\text { Adverse } \\
\text { Events } \\
\text { of BVA }\end{array}$ & Reference \\
\hline $\begin{array}{c}\text { Cold } \\
\text { allodynia }\end{array}$ & $\begin{array}{l}\text { Male Sprague } \\
\text { Dawley rats } \\
(200-220 \mathrm{~g}), \\
\text { oxaliplatin induced } \\
\text { cold allodynia (E, } \\
n=8 / \mathrm{AC}, n=5)\end{array}$ & BV (A. mellifera) & $\begin{array}{l}\text { E: oxaliplatin-induced rats with } \\
\text { DL-pchlorophenylalanine (AR: } \\
\text { intraperitoneal route, dose } 150 \mathrm{mg} / \mathrm{kg}) \\
\text { daily injected for three days and BV } \\
\text { injected once (AR: GV3 acupoint, dose: } \\
0.25 \mathrm{mg} / \mathrm{kg} \text {, BV dissolved in a volume of } \\
0.05 \mathrm{cc} \text { saline) } \\
\text { AC: oxaliplatin-induced rats with saline } \\
\text { (AR: GV3 acupoint, volume: } 0.05 \mathrm{cc} \text { ) and } \\
\text { BV injected once (AR: GV3 acupoint, dose: } \\
0.25 \mathrm{mg} / \mathrm{kg}, \mathrm{BV} \text { dissolved in a volume of } \\
0.05 \mathrm{cc} \text { saline) }\end{array}$ & $\begin{array}{l}\text { Cold allodynia (acetone test) } \\
\text { - } \quad \text { (pre and post treatment of E) } \\
\text { NC } \\
-\quad \begin{array}{l}\text { (pre and post treatment of AC) } \\
p<0.001\end{array}\end{array}$ & $\begin{array}{l}\text { spinal } 5 \text {-HT3 } \\
\text { receptors are } \\
\text { activated to } \\
\text { exert the } \\
\text { anti-allodynic } \\
\text { effect of BVA }\end{array}$ & $\begin{array}{l}\text { Not re- } \\
\text { ported }\end{array}$ & [47] \\
\hline $\begin{array}{c}\text { Cold } \\
\text { allodynia }\end{array}$ & $\begin{array}{c}\text { Male } \\
\text { Sprague-Dawley } \\
\text { rats (average } 200 \mathrm{~g}), \\
\text { oxaliplatin induced } \\
\text { cold allodynia (E, } \\
n=7 / \mathrm{AC} 1 \\
n=8 / \mathrm{AC} 2, n=6)\end{array}$ & BV (A. mellifera) & $\begin{array}{l}\text { - } \quad \text { E: oxaliplatin-induced rats with BV } \\
\text { subcutaneously injected once (AR: GV3 } \\
\text { acupoint, dose: } 0.25 \mathrm{mg} / \mathrm{kg} \text {, BV dissolved } \\
\text { in saline at concentrations of } 0.025 \mathrm{mg} / \mathrm{mL} \text { ) } \\
\text { AC1: E group + mecamylamine } \\
\text { administered once (AR: intraperitoneal } \\
\text { route, dose: } 2 \mathrm{mg} / \mathrm{kg} \text { ) } \\
\text { AC2: E group + atropine administered once } \\
\text { (AR: intraperitoneal route, dose: } 1 \mathrm{mg} / \mathrm{kg} \text { ) }\end{array}$ & 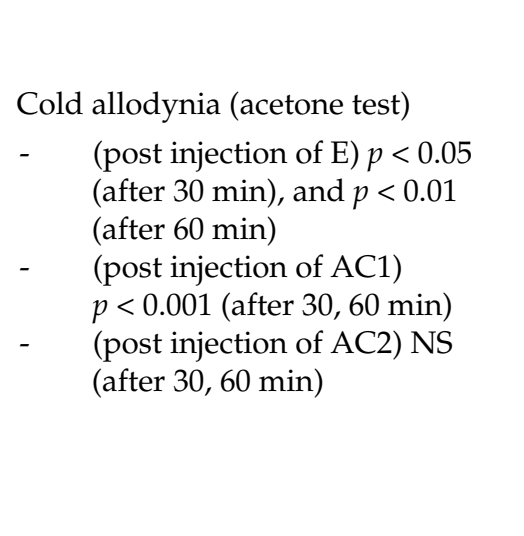 & $\begin{array}{l}\text { BVA treatment } \\
\text { alleviates } \\
\text { oxaliplatin } \\
\text { induced } \\
\text { neuropathic } \\
\text { cold allodynia } \\
\text { in rats via the } \\
\text { activation of } \\
\text { nicotinic } \\
\text { acetylcholine } \\
\text { receptors, } \\
\text { especially } \\
\text { spinal } \alpha 4 \beta 2 \\
\text { receptors. }\end{array}$ & $\begin{array}{l}\text { Not re- } \\
\text { ported }\end{array}$ & [48] \\
\hline
\end{tabular}


Table 5. Cont.

\begin{tabular}{|c|c|c|c|c|c|c|c|}
\hline Disease & $\begin{array}{c}\text { Study Design } \\
\text { (Number of } \\
\text { Patient or Animal } \\
\text { Treated by BV) }\end{array}$ & $\begin{array}{c}\text { Venom/ } \\
\text { Compound/ } \\
\text { (Bee Species) }\end{array}$ & $\begin{array}{c}\text { Intervention } \\
\text { (Dilution Ratio, Amount of Bee Venom Use) }\end{array}$ & Main Results & $\begin{array}{c}\text { Mechanism/ } \\
\text { Molecular Response }\end{array}$ & $\begin{array}{c}\text { Adverse } \\
\text { Events } \\
\text { of BVA }\end{array}$ & Reference \\
\hline $\begin{array}{c}\text { Cold } \\
\text { allodynia }\end{array}$ & $\begin{array}{c}\text { Male } \\
\text { Sprague-Dawley } \\
\text { rats }(210-250 \mathrm{~g}), \\
\text { oxaliplatin induced } \\
\text { cold allodynia (E1, } \\
\begin{array}{c}n=4 / \mathrm{E} 2, n=4 / \mathrm{E} 3, \\
n=4)\end{array}\end{array}$ & $\mathrm{BV}$ (A. mellifera) & $\begin{array}{l}\text { - E: oxaliplatin-induced rats with BV } \\
\text { subcutaneously injected once (AR: GV3 } \\
\text { acupoint, dose: } 1.0 \mathrm{mg} / \mathrm{kg} \text {, BV dissolved in } \\
\text { a volume of } 0.05 \mathrm{cc} \text { saline) } \\
\text { AC1: oxaliplatin-induced rats with BV } \\
\text { subcutaneously injected once (AR: LI11 } \\
\text { acupoint, dose: } 1.0 \mathrm{mg} / \mathrm{kg} \text {, BV dissolved in } \\
\text { a volume of } 0.05 \mathrm{cc} \text { saline) } \\
\text { AC2: oxaliplatin-induced rats with BV } \\
\text { subcutaneously injected once (AR: ST36 } \\
\text { acupoint, dose: } 1.0 \mathrm{mg} / \mathrm{kg}, \mathrm{BV} \text { dissolved in } \\
\text { a volume of } 0.05 \mathrm{cc} \text { saline) }\end{array}$ & $\begin{array}{l}\text { Cold allodynia (acetone test) } \\
\text { - } \quad \text { (pre and post treatment of E) } \\
p<0.05 \text { (after } 1 \mathrm{~h}), \text { and } \\
p<0.001 \text { (after } 2 \mathrm{~h} \text { ) } \\
\text { - } \quad \text { pre and post treatment of } \\
\text { AC1) } p<0.05 \text { (after } 1 \mathrm{~h} \text { ), and } \\
\text { NS (after } 2 \mathrm{~h}) \\
\text { (pre and post treatment of } \\
\text { AC2) } p<0.05 \text { (after } 1 \mathrm{~h}), \text { and } \\
\text { NS (after } 2 \mathrm{~h})\end{array}$ & $\begin{array}{l}\text { BVA alleviates } \\
\text { oxaliplatin } \\
\text { induced cold } \\
\text { allodynia in } \\
\text { rats, at least } \\
\text { partly, through } \\
\text { activation of } \\
\text { the } \\
\text { noradrenergic } \\
\text { system. }\end{array}$ & $\begin{array}{l}\text { Not re- } \\
\text { ported }\end{array}$ & [49] \\
\hline $\begin{array}{c}\text { Cold } \\
\text { allodynia }\end{array}$ & $\begin{array}{l}\text { Male Sprague } \\
\text { Dawley rats } \\
(180-230 \text { g), CCI } \\
\text { induced cold } \\
\text { allodynia }(\mathrm{E} 1, \\
n=6 / \mathrm{E} 2, n=6)\end{array}$ & BV (A. mellifera) & $\begin{array}{l}\text { - } \quad \text { E1: CCI surgery rats with BV injected } \\
\text { subcutaneously twice a day for } 2 \text { weeks } \\
\text { (AR: ST36 acupoint, dose: } 0.25 \mathrm{mg} / \mathrm{kg}, \mathrm{BV} \\
\text { dissolved in a volume of } 50 \mu \mathrm{L} \text { saline) } \\
\text { E2: CCI surgery rats with BV injected } \\
\text { subcutaneously, twice a day for } 2 \text { weeks } \\
\text { (AR: ST36 acupoint, dose: } 2.5 \mathrm{mg} / \mathrm{kg}, \mathrm{BV} \\
\text { dissolved in a volume of } 50 \mu \mathrm{L} \text { saline) } \\
\text { NC: CCI surgery rats with saline injected } \\
\text { subcutaneously, twice a day for } 2 \text { weeks } \\
\text { (AR: ST36 acupoint, dose: not reported) }\end{array}$ & $\begin{array}{l}\text { Cold allodynia (acetone test) } \\
\text { - } \quad \text { (E1 vs. NC) NS (after 15, 30, } \\
45,60,90,120 \text { min) } \\
\text { (E2 vs. NC) } p<0.01 \text { (after 45, } \\
90 \text { min), and } p<0.001 \text { (after } \\
60 \text { min) }\end{array}$ & $\begin{array}{l}\text { BV treatment } \\
\text { could relieve } \\
\text { cold allodynia } \\
\text { via activation of } \\
\text { spinal } \alpha 2- \\
\text { adrenoceptors }\end{array}$ & $\begin{array}{l}\text { Not re- } \\
\text { ported }\end{array}$ & {$[50]$} \\
\hline
\end{tabular}


Table 5. Cont.

\begin{tabular}{|c|c|c|c|c|c|c|c|}
\hline Disease & $\begin{array}{c}\text { Study Design } \\
\text { (Number of } \\
\text { Patient or Animal } \\
\text { Treated by BV) }\end{array}$ & $\begin{array}{c}\text { Venom/ } \\
\text { Compound/ } \\
\text { (Bee Species) }\end{array}$ & $\begin{array}{c}\text { Intervention } \\
\text { (Dilution Ratio, Amount of Bee Venom Use) }\end{array}$ & Main Results & $\begin{array}{c}\text { Mechanism/ } \\
\text { Molecular Response }\end{array}$ & $\begin{array}{c}\text { Adverse } \\
\text { Events } \\
\text { of BVA }\end{array}$ & Reference \\
\hline $\begin{array}{c}\text { Inter- } \\
\text { vertebral } \\
\text { disc } \\
\text { disease } \\
\text { induced } \\
\text { neurologi- } \\
\text { cal } \\
\text { pain }\end{array}$ & $\begin{array}{c}\text { Canines (both } \\
\text { sexes), } \\
\text { intervertebral disc } \\
\text { disease induced } \\
\text { neurological pain } \\
\quad(n=17)\end{array}$ & BV (A. mellifera) & $\begin{array}{l}\text { E: AC group + intervertebral disc disease } \\
\text { induced canines with BV subcutaneously } \\
\text { injected twice a week for } 6 \text { weeks (AR: LI4, } \\
\text { SI3, KI3, ST36, BL23, BL40, GB30, GB34, } \\
\text { LR3, GV1, Baihui, and Ashi acupoints, dose: } \\
5.0 \mathrm{mg} / \mathrm{kg} \text { BV dissolved in a volume of } \\
50 \mu \mathrm{L} \text { saline injected } 0.1 \mathrm{~mL} \text { for each } \\
\text { acupoint) } \\
\text { AC: intervertebral disc disease induced } \\
\text { canines with oral prednisone }(1 \mathrm{mg} / \mathrm{kg}) \\
\text { together with the NSAID carprofen } \\
(2.2 \mathrm{mg} / \mathrm{kg}) \text { for } 7 \text { days. Ranitidine } \\
(2 \mathrm{mg} / \mathrm{kg}) \text { for } 5 \text { or } 7 \text { days was administered } \\
\text { to prevent gastrointestinal disturbance }\end{array}$ & $\begin{array}{l}\text { Neurological pain (myelopathy } \\
\text { scoring system) } \\
\text { - } \quad \text { E vs. AC) } p=0.245 \text { (all } \\
\text { group), } p=0.59 \text { (mild group), } \\
p=0.001 \text { (moderate group), } \\
\text { and } p=0.014 \text { (severe group) }\end{array}$ & Not reported & $\begin{array}{l}\text { Not re- } \\
\text { ported }\end{array}$ & [51] \\
\hline
\end{tabular}

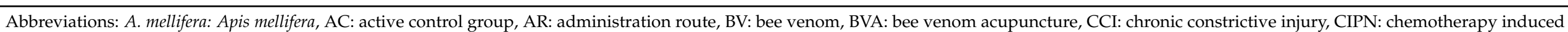

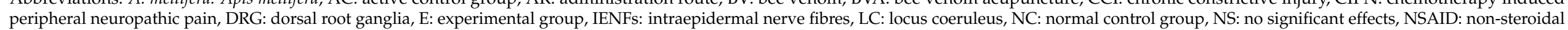

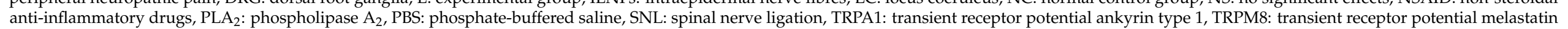
type 8, TRPV1: transient receptor potential vanilloid type 1, WDR: wide dynamic range. 
Table 6. Experimental studies on therapeutic application of bee venom for post-ischaemic pain and prostatic pain.

\begin{tabular}{|c|c|c|c|c|c|c|c|}
\hline Disease & $\begin{array}{c}\text { Study Design } \\
\text { (Number of } \\
\text { Patient or Animal } \\
\text { Treated by BV) }\end{array}$ & $\begin{array}{c}\text { Venom/ } \\
\text { Compound/ } \\
\text { (Bee Species) }\end{array}$ & $\begin{array}{c}\text { Intervention } \\
\text { (Dilution Ratio, Amount of Bee Venom Use) }\end{array}$ & Main Results & $\begin{array}{l}\text { Mechanism/ } \\
\text { Molecular } \\
\text { Response }\end{array}$ & $\begin{array}{l}\text { Adverse } \\
\text { Events } \\
\text { of BVA }\end{array}$ & Reference \\
\hline $\begin{array}{l}\text { Chronic } \\
\text { post- } \\
\text { ischaemic } \\
\text { pain }\end{array}$ & $\begin{array}{c}\text { Male C57/Bl6 } \\
\text { mice }(25-30 \mathrm{~g}), \\
\text { chronic } \\
\text { post-ischaemic } \\
\text { pain model was } \\
\text { induced in mice } \\
\text { with isoflurane by } \\
\text { placing a } \\
\text { tight-fitting } \\
\text { O-ring with a } \\
\text { 5/64-inch internal } \\
\text { diameter around } \\
\text { the left ankle for } \\
3 \mathrm{~h}(n=18)\end{array}$ & $\begin{array}{l}\text { BV }(A . \\
\text { mellifera })\end{array}$ & $\begin{array}{l}\text { - E1: chronic post-ischaemic pain model } \\
\text { mice with BV injected (AR: dorsum of } \\
\text { the ipsilateral hind paw, dose: } \\
1.0 \mathrm{mg} / \mathrm{kg} \text { BV injected once) } \\
\text { E2: chronic post-ischaemic pain model } \\
\text { mice with BV injected (AR: dorsum of } \\
\text { the ipsilateral hind paw, dose: } \\
1.0 \mathrm{mg} / \mathrm{kg} \text { BV injected twice) } \\
\text { E3: chronic post-ischaemic pain model } \\
\text { mice with BV injected (AR: dorsum of } \\
\text { the ipsilateral hind paw, dose: } \\
1.0 \mathrm{mg} / \mathrm{kg} \text { BV injected } 3 \text { times) } \\
\text { NC: chronic post-ischaemic pain model } \\
\text { mice with saline injected once (AR: } \\
\text { dorsum of the ipsilateral hind paw, dose: } \\
\text { same dose of E1 group) }\end{array}$ & $\begin{array}{l}\text { Mechanical allodynia (von Frey } \\
\text { test) } \\
\text { - } \quad \text { (E1 vs. NC) } p<0.05 \text { (after } \\
30 \text { min), } p<0.05 \text { (after } \\
60 \text { min), } p<0.05 \text { (after } \\
90 \text { min), NS (after } 120 \text { min), } \\
\text { NS (after } 180 \text { min), NS (after } \\
240 \text { min), and NS (after } 24 \text { h) } \\
\text { (E2 vs. NC) } p<0.05 \text { (after } \\
30 \text { min), } p<0.05 \text { (after } \\
60 \text { min), } p<0.05 \text { (after } \\
90 \text { min), } p<0.05 \text { (after } \\
120 \text { min), NS (after } 180 \text { min), } \\
\text { NS (after } 240 \text { min), and NS } \\
\text { (after } 24 \text { h) } \\
\text { (E3 vs. NC) } p<0.05 \text { (after } \\
30 \text { min), } p<0.05 \text { (after } \\
60 \text { min), } p<0.05 \text { (after } \\
90 \text { min), } p<0.05 \text { (after } \\
120 \text { min), } p<0.05 \text { (after } \\
180 \text { min), NS (after } 240 \text { min), } \\
\text { and NS (after } 24 \text { h) }\end{array}$ & $\begin{array}{l}\text { Antiallodynic } \\
\text { effect was } \\
\text { objectively } \\
\text { proven } \\
\text { through a } \\
\text { reduction in } \\
\text { the increased } \\
\text { expression of } \\
\text { NK-1 } \\
\text { receptors in } \\
\text { dorsal root } \\
\text { ganglia. }\end{array}$ & $\begin{array}{l}\text { No } \\
\text { adverse } \\
\text { events }\end{array}$ & [52] \\
\hline $\begin{array}{l}\text { Chronic } \\
\text { prostatic } \\
\text { pain }\end{array}$ & $\begin{array}{c}\text { Male } \\
\text { Sprague-Dawley } \\
\text { rats (400-450 g), } \\
\text { complete } \\
\text { Freund's adjuvant } \\
\text { induced chronic } \\
\text { prostatic pain } \\
(n=15)\end{array}$ & $\begin{array}{c}\text { Melittin } \\
(\text { A. mellifera })\end{array}$ & $\begin{array}{l}\text { E: complete Freund's adjuvant induced } \\
\text { rats with melittin injected into both right } \\
\text { and left lobes of the prostate once (dose: } \\
0.05 \mathrm{mg} \text { melittin in a volume of } 0.2 \mathrm{~mL} \\
\text { saline) } \\
\text { - NC: rats with sham operation } \\
\text { - AC: complete Freund's adjuvant } \\
\text { induced rats with saline injected into } \\
\text { both right and left lobes of the prostate } \\
\text { once (volume: } 0.2 \mathrm{~mL})\end{array}$ & $\begin{array}{l}\text { Chronic prostatic pain (Threshold } \\
\text { of mechanical pain) } \\
\text { - } \quad \text { (E vs. NC) NS (after } 0 \text { day), } \\
\text { NS (after } 6 \text { days), } p<0.01 \\
\text { (after } 12 \text { days), and } p<0.01 \\
\text { (after } 18 \text { days) } \\
\text { ( } \mathrm{E} \text { vs. AC) NS (after } 0 \text { day), } \\
\text { NS (after } 6 \text { days), NS (after } \\
12 \text { days), and } p<0.05 \text { (after } \\
18 \text { days) }\end{array}$ & $\begin{array}{l}\text { Melittin could } \\
\text { alleviate } \\
\text { complete } \\
\text { Freund's } \\
\text { adjuvant } \\
\text { induced } \\
\text { prostatitis pain } \\
\text { in rat by } \\
\text { suppressing } \\
\text { cyclooxygenase- } \\
2 \text { expression }\end{array}$ & $\begin{array}{l}\text { Not re- } \\
\text { ported }\end{array}$ & [53] \\
\hline
\end{tabular}

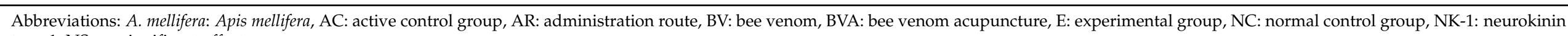
type 1, NS: no significant effects. 


\section{Summary of the Previous Review}

Chen et al. [13] collected and reviewed articles on the nociceptive and anti-nociceptive effects of BV therapy published before 2010. The main components of BV are peptide constituents such as enzymes (e.g., phospholipase A2 ( $\mathrm{PLA}_{2}$ ) and hyaluronidase), apamin, melittin, mast cell degranulating peptide, and adolapin. Among them, melittin (40-60\%), adolescent $(1 \%)$, and $\mathrm{PLA}_{2}(12 \%)$ were reported to exert antinociceptive effects. Mast-cell degranulating peptide $(2-3 \%)$ and hyaluronidase $(<3 \%)$ produced histamines associated with allergic reactions to BV therapies. Experimental human and animal studies conducted on BV-induced pain, inflammation, and allergic responses were examined. Honeybee stings cause hyperalgesia, long-lasting pain, and local inflammation, suggesting that live bee sting therapy may cause such adverse events. Additionally, a significant number of papers have been conducted in the past decade, highlighting the anti-inflammatory and anti-nociceptive effects of BV therapies and components of BV. In clinical evidence, the effects of BVA for treating pain (e.g., pain of osteoarthritis of the knee, and musculoskeletal pain) have been reported. The anti-inflammatory and anti-nociceptive effects of BVA have been demonstrated more comprehensively in various animal pain models, including those pertaining to the study of adjuvants (rheumatoid arthritis model), collagen type-II (inflammatory model), carrageenan (inflammatory model), and chronic constriction injury (neuropathic pain model).

\section{Therapeutic Effects of BVA in Pain}

\subsection{Musculoskeletal Pain}

3.1.1. Clinical Studies

Shoulder Pain

In a systematic review involving seven randomized controlled trails (RCTs) [14], shoulder pain was observed to be significantly lower for BVA (administration route (AR): GB21, LI11, LI15, LI16, SI3, SI9, SI10, SI11, TE14, and UE12 acupoints) plus conventional treatment $(\mathrm{CT})$ than that reported for saline injection plus $\mathrm{CT}$ on the pain rating scale (PRS) $(p=0.009)$ and visual analogue scale (VAS) $(p=0.03)$. The BVA concentrations of the collected RCTs ranged from $0.03-0.5 \mathrm{mg} / \mathrm{mL}$, the total treatment sessions were 6-16 times, and the total volume ranged from $0.6-14.8 \mathrm{~mL}$. Among the 45 patients who underwent BVA, 2 presented with pain, 8 exhibited pruritus, 3 experienced a burning sensation, 30 presented with pruritus/local swelling/redness, and 1 showed mild, generalised swelling/aching. This study suggests that shoulder pain can be treated considering the BVA procedure in addition to $\mathrm{CT}$, but standardisation of the dose and the total number of procedures should be considered.

In an RCT reported by An et al. [15], a total of 101 patients received either conventional or needle-free BVA for examination of the therapeutic effects of BVA on myofascial shoulder pain. BV was administered to the GB21 acupoint for both groups, and shoulder pain was significantly improved by the treatment in both groups. The concentration of BV was not reported, and a total of two treatment sessions were performed, with a total volume of $2 \mathrm{~mL}$. Adverse events (AEs) were less frequently reported in the needle-free group (inflammation, 1; purpura, 2; headache, 2; and redness, 1) than in the BVA (fatigue, 1; purpura, 1; oedema, 2; headache, 1; cold, 1; itching, 16; and redness, 14) groups.

Two RCTs [16,17] reported the assessment of BVA for adhesive capsulitis pain. One study [16] recruited 68 patients into three groups: BVA group1 $(0.1 \mathrm{mg} / \mathrm{mL}$ BVA plus physiotherapy), BVA group2 ( $0.03 \mathrm{mg} / \mathrm{mL}$ BVA plus physiotherapy), and control group (saline injection plus physiotherapy). Both BVA groups injected BV into GB21, LI15, LI16, SI11, and TE14 acupoints. Compared to the control group, the BVA group1 presented significant improvements in the shoulder pain and disability index (SPADI) at 8 days $(p=0.025)$ and 12 days $(p=0.014)$, and VAS score at rest at the eighth week $(p=0.048)$, during the motion score at the 12th week $(p=0.029)$. Both BVA groups received 16 sessions of treatment, and the total BV volume was $14.8 \mathrm{~mL}$. A one-year follow-up analysis of a previously reported RCT [17] was conducted using a tele-interview. The SPADI scores at 
one-year statistically differed between the $0.1 \mathrm{mg} / \mathrm{mL}$ BVA plus physiotherapy group and the saline injection plus physiotherapy group $(p=0.043)$. The therapeutic effect on adhesive capsulitis pain was partially confirmed using BVA at a concentration of $0.1 \mathrm{mg} / \mathrm{mL}$ rather than at a concentration of $0.03 \mathrm{mg} / \mathrm{mL}$, and minor AEs were confirmed.

\section{Low Back Pain}

Two RCTs [18,19] and one retrospective study [20] examined the treatment effect of BVA on low back pain. In a RCT reported by Seo et al. [18], a total of 54 subjects received either BVA (AR: BL23, BL24, BL25, GB30, GV3, GV4, and GV5 acupoints) plus non-steroidal anti-inflammatory drugs (NSAIDs) or saline injection plus NSAID for six treatments over a period of 3 weeks. The BVA plus NSAID group showed a significant reduction in chronic low back pain $(p=0.0486)$ compared to the control group, as measured by using VAS. The BVA concentration was $0.05 \mathrm{mg} / \mathrm{mL}$, and the total volume was $28 \mathrm{~mL}$ across six treatment sessions. Among the 27 subjects treated with BVA, itching/sensation was observed in four patients, headache occurred in one patient, and generalised myalgia occurred in one patient. Shin et al. [19] randomly divided 60 patients into BVA (AR: BL23, BL24, and BL25 acupoints) and saline injection groups. In VAS used for pain intensity, the BVA group showed a statistically significant difference $(p=0.0087)$ compared to saline injection. The BVA concentration was $0.05 \mathrm{mg} / \mathrm{mL}$, the total treatment sessions were eight times, and the total volume was $4.8 \mathrm{~mL}$. Among the 30 subjects treated with BVA, 15 presented with pruritus, 5 experienced skin flares, 4 showed oedema, and 2 presented with skin rash.

In a retrospective observational study of BVA (AR: 4-5 acupoints around the lumbar spine) for low back pain of intervertebral disc herniation [20], a total of 524 participants who received non-surgical complementary and alternative medicine treatment (BVA, acupuncture, herbal medicine, and chuna) exhibited a numeral rating scale (NRS) score of 3.18-2.29 (95\% confidence interval [CI], 2.99-3.38). The BVA concentration was $0.1 \mathrm{mg} / \mathrm{mL}$, the total treatment sessions were $2.3 \pm 1.8$ times, and the total volume was not reported. Allergic reactions caused by BV were observed in eight patients. The clinical trial of Shin et al. [20] is not a single treatment of BVA but suggests that BVA is clinically used for low back pain. Additionally, in the two RCTs [18,19], the BVA concentration was the same at $0.05 \mathrm{mg} / \mathrm{mL}$, but the total volume was approximately five times different. Standardisation of treatment sessions and dosage is required.

\subsection{Inflammatory Pain \\ 3.2.1. Clinical Studies}

BVA was used in 361 patients with knee osteoarthritis pain [21], and subjects were randomly assigned to the BVA or histamine injection groups. The BVA group injected BV into BL40, BL19, BL 21, BL 23, BL 25, BL27, ST34, five on each knee (knee top), eye-one medial, and eye-two lateral. The control group received the same acupoint injection as the BVA group. The BVA group showed significant improvement in the Western Ontario and McMaster Universities Osteoarthritis index (WOMAC) pain score after 12 weeks (95\% CI, 0.3-2.0, $p=0.0010$ ). BV powder $1 \mathrm{mg}$ and $1 \mathrm{~mL}$ of $0.5 \%$ lidocaine were mixed, and 12 treatment sessions were conducted with a total dosage of $17.1 \mathrm{~mL}$. Among the patients treated with BVA, AEs occurred in 177 patients and injection site AEs occurred in 15 patients.

\subsubsection{Experimental Studies}

Two experimental studies $[22,23]$ have investigated arthritis pain in a mouse model. BV (1 mg/200 g) showed anti-nociceptive activity in Freund's complete adjuvant (FCA) and collagen type-II-induced rats with arthritis pain [22]. For FCA-induced rats, the dorsal flexion test score was significantly reduced in BVA (AR: right hind paw, dose: $1 \mathrm{mg} / 200 \mathrm{~g}$ ) and conventional drug (indomethacin, $2 \mathrm{mg} / \mathrm{kg}$ ) administration in comparison with the saline injection group. In the collagen type-II-induced rat model, BVA and indomethacin administered groups revealed a decrease in the dorsal flexion score compared 
to the saline-injected group. Yamasaki et al. [23] compared the effectiveness of BVA (AR: dorsal route, dose: $0.25 \mathrm{mg} / \mathrm{kg}$ in a volume of $50 \mu \mathrm{L}$ ) and methotrexate (AR: dorsal route, dose: $0.3 \mathrm{mg} / \mathrm{kg}$ in a volume of $300 \mu \mathrm{L}$ ), the most commonly utilized diseasemodifying anti-rheumatic drug in rats with collagen type-II-induced arthritis pain. The value of the mechanical threshold of hyperalgesia in healthy rats was $46 \pm 2$. The reduced mechanical threshold of hyperalgesia persisted in all collagen type-II-induced rat groups, but with amelioration in relation to untreated arthritic rats $(11 \pm 1.30)$, in the methotrexate administration group ( $22 \pm 1.70)$, and in the group subjected to a combination of BVA and methotrexate administration $(23.5 \pm 2.20)$.

Two experimental studies [24,25] have focused on formalin-induced inflammatory pain. Kang et al. [24] assessed the anti-nociceptive effect of BVA in ST36 acupoint in formalin-induced mice with inflammatory pain during the first and second round of the formalin test. Although injection of a low dose of $0.08 \mathrm{mg} / \mathrm{kg}$ BVA did not improve formalin-induced pain behaviour, a higher dose of $0.8 \mathrm{mg} / \mathrm{kg}$ BVA significantly improved pain responses in the second round of the formalin test compared to that of the saline injection group. Experiments were performed for assessing formalin-induced inflammatory pain responses elicited during the early and late round of the formalin test [25]. The results of BVA (AR: ST36 acupoint, dose: $0.8 \mathrm{mg} / \mathrm{kg}$ ) and BVA plus hydroxydopamine (AR: intraperitoneal route, volume: $100 \mu \mathrm{L}$ ) showed significant improvements on formalininduced pain behaviour as compared with the saline injection group during late round of the formalin test.

Huh et al. [26] investigated the analgesic effects of BVA in a rat model of collagen typeII-induced osteoarthritis pain. The BVA (AR: ST36 acupoint, dose: $1.0 \mathrm{mg} / \mathrm{kg}$ ) resulted in a more significant pain reduction effect than the non-acupoint BVA (AR: intraperitoneal route, dose: $1.0 \mathrm{mg} / \mathrm{kg}$ ) and no treatment groups. Pain-related response was more improved by treatment with $1 \mathrm{mg} / \mathrm{kg}$ BVA (AR: ST36 acupoint) than that observed with $2 \mathrm{mg} / \mathrm{kg}$ BVA (AR: ST36 acupoint).

\subsection{Neuropathic Pain}

\subsubsection{Clinical Studies}

\section{Post-Stroke Pain}

A meta-analysis of two RCTs [27] showed that post-stroke shoulder pain was significantly lower in the BVA group (AR: EX-UE70, GB21, LI11, LI15, SI3, SI9, SI10, SI11, and TE14 acupoints) than that in the saline injection group in VAS ( $p=0.02)$. The BVA concentration was $0.01-0.5 \mathrm{mg} / \mathrm{mL}$, the total treatment sessions were 6-12 times, and the total volume was 0.9-13.5 mL. A single-blind RCT [28] investigated the central post-stroke pain. BVA or saline was administered into GB21, GB31, GB39, LI11, LI15, and ST36 acupoints. After three weeks, the VAS score improved more in the BVA group than that in the control group $(p=0.009)$. The concentration of BV was not reported, but the total volume was $1.8 \mathrm{~mL}$ over six treatment sessions. No AEs occurred in the BVA group. These studies indicate that BVA is effective for treating post-stroke pain and musculoskeletal pain.

\section{Chemotherapy-Induced Peripheral Neuropathic Pain}

Two case studies [29,30] focused on BVA for chemotherapy-induced peripheral neuropathic (CIPN) pain. Park et al. [29] treated four CIPN pain patients with BVA $(0.1 \mathrm{mg} / \mathrm{mL}$, three sessions of $4.8 \mathrm{~mL}$ ) on $\mathrm{Ba}$ Xie and Ba Feng acupoints (total of 16 points located between all 5 fingers and toes). The average VAS score decreased from 8.75 to 2.75, and AEs were not observed. Yoon et al. [30] treated 11 patients with CIPN pain using BVA $(0.1 \mathrm{mg} / \mathrm{mL})$ on GB39, LI4, LV3, and SJ5 acupoints. The mean VAS score significantly decreased 3 weeks after the final treatment $(p<0.01)$ and continued to decrease after 6 weeks $(p<0.05)$. Minor AEs (swelling and itchiness, 2; mild fever, 1) were reported in the study. Regarding pain reduction mechanisms, Yoon et al. [30] reported that BVA may demonstrate functions based on mechanisms similar to those of adrenergic reuptake inhibitors or norepinephrine reuptake inhibitors. Two studies were conducted at the East-West Cancer Center of Dunsan 
Oriental Hospital of Daejeon University using the same concentration, dose and volume. This study will be developed as a dose-response evaluation to further establish the safety and response prior to clinical trial.

\section{Complex Regional Pain Syndrome and Refractory Postherpetic Neuralgia}

One patient diagnosed with complex regional pain syndrome after toe surgery received BVA at the GB43 acupoint [31]. After treatment, the worst level of the numeral rating scale (NRS) was reduced from 8 to 0 , the usual level of NRS was reduced from 5 to 0 , and the best level of NRS was reduced from 3 to 0 . BVA concentration was not reported, the total number of treatment sessions was 14 , and the volume was $4.55 \mathrm{~mL}$. No side effects related to BVA occurred. In another study [32], one patient with refractory postherpetic neuralgia was treated with BVA $(0.03 \mathrm{mg} / \mathrm{mL})$ once a week for 4 weeks. The patient's NRS score decreased from 8 to 2, and itchiness at the site of injection occurred for 2 days following treatment.

\subsubsection{Experimental Studies \\ Mechanical Allodynia}

Many studies have studied whether BVA could be used to reduce mechanical allodynia, a representative symptom of neuropathic pain. Two experimental studies [33,34] investigated the use of BVA in spinal nerve ligation (SNL)-induced mechanical allodynia. In a study reported by Woo et al. [33], SNL was conducted in rats to induce neuropathic pain. SNL-induced mechanical allodynia rats were injected in the intraperitoneal route with $0.2 \mathrm{mg} / \mathrm{kg} \mathrm{PLA}_{2}$ or $0.2 \mathrm{mg} / \mathrm{kg}$ phosphate-buffered saline (PBS). PLA 2 is a main component of BV, consisting of $10 \%-12 \%$ of dry BV $[54,55]$. The PLA $_{2}$ group showed a significant decrease in mechanical allodynia at 2 days $(p<0.001)$ compared to the control group. Perineural pre-treatment of the L5 and L6 spinal nerves with BVA significantly improved mechanical allodynia in SNL-induced rats [34]. The mechanical hind paw withdrawal threshold was more improved in rats treated with $0.05 \mathrm{mg} / \mathrm{kg}$ and $0.1 \mathrm{mg} / \mathrm{kg}$ BVA on days $3,5,7,9$, and 13 compared to the saline treated control group.

Two animal studies $[35,36]$ focused on the effectiveness of BVA on paclitaxel-induced mechanical allodynia. A mechanical allodynia model was induced in rats treated with paclitaxel, a chemotherapy drug for various tumours [35]. Paclitaxel-induced rats were injected with $1.0 \mathrm{mg} / \mathrm{kg}$ BVA or PBS at the right side of the ST6 acupoint. The BVA group showed a significant effect in mechanical allodynia at $1 \mathrm{~h}(p<0.05)$ and $2 \mathrm{~h}(p<0.01)$ after injection. Li et al. [36] investigated the combined effects of BVA (AR: ST36 acupoint, dose: $1.0 \mathrm{mg} / \mathrm{kg}$, BV dissolved in a volume of $20 \mu \mathrm{L}$ PBS) and venlafaxine (AR: oral route, dose: $40 \mathrm{mg} / \mathrm{kg}$, venlafaxine dissolved in a saline) on mechanical allodynia induced by paclitaxel, a primary chemotherapeutic agent utilized to treat various solid malignancies. Co-treatment with BVA and venlafaxine produced a longer-lasting anti-allodynic effect (180 $\mathrm{min}$ ) than that induced by BVA (effects continued for $120 \mathrm{~min}$ ) or venlafaxine (effects continued for $60 \mathrm{~min})$.

Several studies [37-43] have examined the anti-allodynic effect of BVA on oxaliplatininduced mechanical allodynia. Kim et al. [37] assigned 32 oxaliplatin-induced mechanical allodynia model mice into four groups. Oxaliplatin is a chemotherapeutic drug used to treat colorectal cancer that causes severe peripheral neuropathy. Both morphine (AR: intraperitoneal route, dose: $2.0 \mathrm{mg} / \mathrm{kg}$ in a volume of $0.2 \mathrm{~mL}$ ) with a saline injection and BVA (AR: ST36 acupoint, dose: $1.0 \mathrm{mg} / \mathrm{kg}$ was injected in a volume $20 \mu \mathrm{L}$ ) with a saline injection exerted significant effects that continued $120 \mathrm{~min}$ after the treatment. However, these analgesic effects were less significant than the effect of the combination of BVA and morphine, which lasted $180 \mathrm{~min}$. These results indicate that combination treatment of BVA and morphine is effective in oxaliplatin-induced mechanical allodynia. Li et al. [38] investigated the effects of BV (AR: not reported, dose: $1.0 \mathrm{mg} / \mathrm{kg}$, BV dissolved in a PBS) and PLA 2 (AR: not reported, dose: $0.2 \mathrm{mg} / \mathrm{kg}$, $\mathrm{PLA}_{2}$ dissolved in a PBS) on oxaliplatin-induced mechanical allodynia in mice. BVA significantly reduced mechanical 
allodynia from day 2 and the effect continued until day 4 . The PLA 2 mice group showed a significant improvement in mechanical allodynia from day 2 and the effects lasted up to day 5 . In the study reported by $\mathrm{Li}$ et al. [39], PLA 2 (AR: intraperitoneal route, dose: $0.2 \mathrm{mg} / \mathrm{kg}$ ) pre-treatment significantly improved mechanical allodynia on day 3 . These results suggest that $\mathrm{PLA}_{2}$ has the potential to be used in the prevention of oxaliplatininduced mechanical allodynia. Yoon et al. [40] evaluated oxaliplatin-induced mechanical allodynia in the hind paws of mice and examined the effect of BVA on mechanical allodynia. No significant differences were observed between the paw withdrawal threshold values before and after injection in the saline (AR: ST36 acupoint, dose: not reported) and the BVA-Back (AR: back point, dose: $0.1 \mathrm{mg} / \mathrm{kg}$, BV dissolved in a volume of $20 \mu \mathrm{L}$ saline) groups in hind paws. One hour after BVA (AR: ST36 acupoint, dose: $0.1 \mathrm{mg} / \mathrm{kg}$, BV dissolved in a volume of $20 \mu \mathrm{L}$ saline) treatment, significant increases in paw withdrawal threshold values were observed in the right hind paw compared with the saline injection group. Yeo et al. [41] investigated BVA (AR: ST36 acupoint, dose: $0.1 \mathrm{mg} / \mathrm{kg}$, BV dissolved in a volume of $20 \mu \mathrm{L}$ saline) in oxaliplatin-induced mice with mechanical allodynia. The results showed a significant long-lasting analgesic effect of repetitive $0.1 \mathrm{mg} / \mathrm{kg}$ BVA on the paw withdrawal threshold before and after subjection to BVA compared with the saline injection group (AR: ST36 acupoint, dose: not reported). In contrast, no significant differences were shown in the paw withdrawal threshold before and after injection in the non-acupoint BVA group (AR: back point, dose: $0.1 \mathrm{mg} / \mathrm{kg}$, BV dissolved in a volume of $20 \mu \mathrm{L}$ saline). Choi et al. [42] investigated the anti-nociceptive effect of melittin on oxaliplatin-induced mechanical allodynia. The mechanical allodynia was significantly reduced $30 \mathrm{~min}$ after melittin injection $(0.5 \mathrm{mg} / \mathrm{kg})$ on ST36 acupoint. In a study reported by Lee et al. [43], single subcutaneous administration of BVA $(0.1 \mathrm{mg} / \mathrm{kg}$ and $1 \mathrm{mg} / \mathrm{kg})$ at ST36 acupoint reduced mechanical allodynia induced by oxaliplatin in rats. Mechanical allodynia evaluated using von Frey tests was significantly improved 30 min after injection with $0.1 \mathrm{mg} / \mathrm{kg}$ and $1 \mathrm{mg} / \mathrm{kg}$ of BV compared to the control (post-injection; $p<0.01$ and $p<0.001$ for $0.1 \mathrm{mg} / \mathrm{kg} \mathrm{BV}$ and $1 \mathrm{mg} / \mathrm{kg} \mathrm{BV}$, respectively).

Three animal studies [44-46] investigated a neuropathic pain rat model induced by chronic constrictive injury (CCI), spinal cord injury (SCI), and vincristine. In the study reported by Kang et al. [44], BV (AR: ST36 acupoint, dose: $0.25 \mathrm{mg} / \mathrm{kg}$, BV dissolved in a volume of $50 \mu \mathrm{L}$ saline) was injected subcutaneously twice a day for 2 weeks from 15 days after CCI surgery. Repeated administration of BV significantly reduced the paw withdrawal frequency from 4 days and continued for 13 days compared with the saline injection group (AR: ST36 acupoint, dose: not reported). Kang et al. [45] examined the effect of repetitive BVA $(0.25 \mathrm{mg} / \mathrm{kg})$ on SCI-induced mechanical allodynia. Repetitive BVA (AR: ST36 acupoint, dose: $0.25 \mathrm{mg} / \mathrm{kg}$, BV dissolved in a volume of $50 \mu \mathrm{L}$ saline) significantly increased in the paw withdrawal threshold in the ipsilateral hind paw on day 3 and 5 after treatment. Peripheral neuropathy was induced in rats with vincristine, a chemotherapy drug for solid and hematologic cancers [46]. $1.0 \mathrm{mg} / \mathrm{kg}$ BVA, $0.5 \mathrm{mg} / \mathrm{kg}$ melittin, $0.12 \mathrm{mg} / \mathrm{kg} \mathrm{PLA}$, or saline was subcutaneously injected at the ST36 acupoint. Compared with the saline group, paw withdrawal was significantly decreased after BVA $(1 \mathrm{mg} / \mathrm{kg})$, which lasted until $60 \mathrm{~min}$. The effect of melittin injection was significant and continued for $120 \mathrm{~min}$. PLA 2 injection showed no significant effect after treatment.

\section{Cold Allodynia}

Most animal studies [37-39,42,43,47-49] have examined the analgesic effect of BVA on oxaliplatin-induced cold allodynia. In a study reported by Kim et al. [37], a cold allodynia model was induced in mice treated with oxaliplatin, a chemotherapeutic drug for cancer. BVA $(1.0 \mathrm{mg} / \mathrm{kg}$ was injected into ST36 acupoint in a volume $20 \mu \mathrm{L})$ with saline injection (AR: ST36 acupoint, volume: $0.2 \mathrm{~mL}$ ) exerted a significant effect at the beginning $(60 \mathrm{~min})$, but its anti-allodynic effect could not be observed at $180 \mathrm{~min}$ after injection. Morphine (AR: intraperitoneal route, dose: $2.0 \mathrm{mg} / \mathrm{kg}$ in a volume of $0.2 \mathrm{~mL}$ ) with saline injection (AR: intraperitoneal route, volume: $0.2 \mathrm{~mL}$ ) did not exert any significant analgesic effect. 
The combination of BVA and morphine showed a significant and longer effect than that observed with treatment using either BVA or morphine alone. The cold allodynia model was induced in male mice treated with oxaliplatin [38]. BVA (AR: not reported, dose: $1.0 \mathrm{mg} / \mathrm{kg}$, BV dissolved in a PBS) for five consecutive days significantly improved cold allodynia from day 3 and the effect continued up to day 5. PLA 2 injection (AR: not reported, dose: $0.2 \mathrm{mg} / \mathrm{kg}, \mathrm{PLA}_{2}$ dissolved in a PBS) significantly reduced cold allodynia from day 1 , and this effect persisted for at least six days. These results indicate that PLA 2 injection significantly alleviates oxaliplatin-induced cold allodynia. Li et al. [39] investigated the preventive effects of $\mathrm{PLA}_{2}$ on oxaliplatin-induced cold allodynia. Pre-treatment with $\mathrm{PLA}_{2}$ (AR: intraperitoneal route, dose: $0.2 \mathrm{mg} / \mathrm{kg}$ ) once a day for five days significantly improved cold allodynia from days 3 to 7 . Lee et al. [47] evaluated the effect of 5-HT depletion (AR: intraperitoneal route, dose $150 \mathrm{mg} / \mathrm{kg}$ ) on the reduction effect of BVA (AR: GV3 acupoint, dose: $0.25 \mathrm{mg} / \mathrm{kg}$, BV dissolved in a volume of $0.05 \mathrm{cc}$ saline) on cold allodynia in oxaliplatin-treated mice. The control group (0.05 cc saline pre-treatment on GV3 acupoint) showed a significant improvement in tail withdrawal latency after $0.25 \mathrm{mg} / \mathrm{kg} \mathrm{BVA}(p<0.001)$, whereas the depletion of 5 -HT by DL-pchlorophenylalanine $(150 \mathrm{mg} / \mathrm{kg})$ pre-treatment blocked the anti-allodynic effect of $0.25 \mathrm{mg} / \mathrm{kg}$ BVA $(p>0.05)$. These results indicate that the serotonergic system plays a main role in mediating the antinociceptive effect of BVA on oxaliplatin-induced cold allodynia. The melittin injection on ST36 acupoint reduced cold allodynia caused by oxaliplatin injection [42]. Cold allodynia was significantly improved after melittin injection $(0.5 \mathrm{mg} / \mathrm{kg})$. In contrast, saline injection (AR: ST36 acupoint, dose: not reported) did not exert any measurable effects on cold allodynia. Lee et al. [43] assessed the effects of $0.1 \mathrm{mg} / \mathrm{kg} \mathrm{BVA}, 1.0 \mathrm{mg} / \mathrm{kg}$ BVA, or PBS, subcutaneously injected at the ST36 acupoint on oxaliplatin-induced cold allodynia model rats. The results showed that only BVA $(1 \mathrm{mg} / \mathrm{kg})$ showed a significant analgesic effect $(p<0.05)$ for cold allodynia, $30 \mathrm{~min}$ after BV injection. Yoon et al. [48] showed the relieving effect of BVA on oxaliplatin-induced cold allodynia. Behavioural tests for cold allodynia were conducted before oxaliplatin injection, $30 \mathrm{~min}$ after injection, and $60 \mathrm{~min}$ after treatment. The significant relieving effect of BVA (AR: GV3 acupoint, dose: $0.25 \mathrm{mg} / \mathrm{kg}$, BV dissolved in saline at concentrations of $0.025 \mathrm{mg} / \mathrm{mL}$ ) on cold allodynia was blocked by pre-treatment with $2 \mathrm{mg} / \mathrm{kg}$ mecamylamine (a non-selective nicotinic receptor antagonist), but not by $1 \mathrm{mg} / \mathrm{kg}$ atropine (a non-selective muscarinic receptor antagonist). Lim et al. [49] investigated whether BVA relieved oxaliplatin-induced cold allodynia. BVA $(1.0 \mathrm{mg} / \mathrm{kg})$ at GV3, LI11, and ST36 acupoints significantly improved cold allodynia; the anti-allodynic effect lasted for $2 \mathrm{~h}$ in the GV3 group and persisted for $1 \mathrm{~h}$ in the other groups (LI11 and ST36 acupoints).

In a study reported by Koh et al. [34], a cold allodynia model was induced in male rats with SNL. The cold allodynia in the both $0.1 \mathrm{mg} / \mathrm{kg}$ and $0.05 \mathrm{mg} / \mathrm{kg}$ BVA groups (AR: peri-neural sheath of the L5 and L6 spinal nerves) was observed on the first day of the acetone test and effects lasted until the completion of the study, with effects continuing for more than 29 days. The $0.1 \mathrm{mg} / \mathrm{kg}$ BV group showed effects that were significantly longer in the cold plate test compared with the $0.05 \mathrm{mg} / \mathrm{kg}$ BV group throughout the study duration.

Three animal studies $[36,46,50]$ investigated a neuropathic pain mouse model induced by paclitaxel, vincristine, and chronic constriction injury (CCI). Li et al. [36] assessed the analgesic effects of paclitaxel on cold allodynia. The co-administration of BVA (AR: ST36 acupoint, dose: $1.0 \mathrm{mg} / \mathrm{kg}$, BV dissolved in a volume of $20 \mu \mathrm{L}$ PBS) and venlafaxine (AR: oral route, dose: $40 \mathrm{mg} / \mathrm{kg}$, venlafaxine dissolved in a saline) significantly reduced licking and shaking frequencies (cold allodynia) compared to the BVA group from $60 \mathrm{~min}$, and effects lasted up to $180 \mathrm{~min}$. Li et al. [46] examined the effects of BVA in alleviating peripheral neuropathic pain induced in rats with vincristine. Behavioural tests were conducted for the ipsilateral hind paw before $1.0 \mathrm{mg} / \mathrm{kg}$ BVA, $0.5 \mathrm{mg} / \mathrm{kg}$ melittin, $0.12 \mathrm{mg} / \mathrm{kg} \mathrm{PLA}_{2}$, or saline injection on the ST36 acupoint and were assessed at 30,60, and $120 \mathrm{~min}$ after injection. Among the injected components, BVA significantly decreased cold allodynia in 
the acetone test, and the effects lasted up to $60 \mathrm{~min}$. Kang et al. [50] investigated whether $0.25 \mathrm{mg} / \mathrm{kg}$ BVA or $2.5 \mathrm{mg} / \mathrm{kg}$ BVA reduced cold allodynia in sciatic nerve CCI rats. The single-high-dose BVA ( $2.5 \mathrm{mg} / \mathrm{kg}$, twice a day for 2 weeks) on ST36 acupoint administered after CCI significantly improved the CCI-induced paw withdrawal frequency increase from $45 \mathrm{~min}$ to 90 min compared to the saline injection group (AR: ST36 acupoint, dose: not reported). However, the mice injected with the single-low-dose BVA $(0.25 \mathrm{mg} / \mathrm{kg})$ on the ST36 acupoint did not demonstrate an effect at any time point.

Intervertebral Disc Disease-Induced Neurological Pain

Intervertebral disc disease (IDD) is a spinal disorder that causes neurological pain and dysfunction [51]. A single-blind RCT involving 36 adult canines with IDD pain was divided into two groups. The control group received CT with oral prednisone $(1 \mathrm{mg} / \mathrm{kg})$ together with the nonsteroidal anti-inflammatory drug (NSAID) carprofen $(2.2 \mathrm{mg} / \mathrm{kg})$ and ranitidine $(2 \mathrm{mg} / \mathrm{kg})$. The experimental group was subcutaneously injected with BV (5 mg/kg) into the LI4, SI3, KI3, ST36, BL23, BL40, GB30, GB34, LR3, GV1, Baihui, and Ashi acupoints, and also received control treatment. The myelopathy scoring system was significantly improved in the moderate $(p=0.001)$ and severe groups $(p=0.014)$ but was not significantly reduced in the mild group $(p=0.59)$. The results suggest that BVA plus $\mathrm{CT}$ is more effective for IDD pain treatment than CT alone.

\subsection{Experimental Studies of Post-Ischaemic Pain}

In the study reported by Lee et al. [52], a chronic post-ischaemic pain model was induced in male C57/Bl6 mice with isoflurane by placing a tight-fitting O-ring at the left ankle for $3 \mathrm{~h}$. The significant effect persisted for different durations in the BVA group (AR: dorsum of the ipsilateral hind paw, dose: $1.0 \mathrm{mg} / \mathrm{kg}$ ) compared with the saline injection group: $90 \mathrm{~min}$ for the single BV injection group, $120 \mathrm{~min}$ for the double BV injection group, and $180 \mathrm{~min}$ for the triple BV injection group. The results indicate that repeated BV injections amplify the anti-allodynic effect in mice with chronic post-ischaemic pain.

\subsection{Experimental Studies of Prostatic Pain}

Complete Freund's adjuvant (CFA)-induced prostate pain model rats were injected with melittin or saline into both the right and left lobes of the prostate, and one rat group received a sham operation [53]. Melittin is a major biological component of BV, accounting for $40 \%-60 \%$ of its complete dry weight [36]. Compared to the sham operation group, the melittin group (dose: $0.05 \mathrm{mg}$ melittin in a volume of $0.2 \mathrm{~mL}$ saline) showed significant effects in the threshold of mechanical pain at 12 days $(p<0.01)$ and 18 days $(p<0.01)$. The melittin group showed a significant reduction in prostatic pain at 18 days $(p<0.05)$ compared with the saline injection group (volume: $0.2 \mathrm{~mL}$ ). This study suggests that melittin may be used as a treatment for chronic prostatic pain.

\section{Therapeutic Mechanisms of BV on Pain Management}

The therapeutic mechanisms of BVA reported in experimental studies and clinical trials are as follows.

(1) In cases of musculoskeletal pain, seven clinical studies [14-20] did not report the analgesic mechanism of BVA.

(2) In cases of inflammatory pain, interleukin-6 (IL-6) and tumour necrosis factor- $\alpha$ (TNF- $\alpha$ ) levels were found to be decreased by BVA (Species: Apis dorsata (A. dorsata), AR: oral route, dose: $2.0 \mathrm{mg} / \mathrm{kg}$ ) in a collagen type-II induced arthritis pain model [22]. BVA (Species: Apis mellifera (A. mellifera), AR: dorsal route, dose: $0.25 \mathrm{mg} / \mathrm{kg}$ in a volume of $50 \mu \mathrm{L}$ ) restores altered activity of basic (APN) in synovial fluid (SF), soluble fraction (S) from synovial tissue (ST), and dipeptidyl peptidase IV (DPPIV) in solubilised membrane-bound fraction (M) from peripheral blood mononuclear cells (PBMCs) [23]. BVA attenuates body mass (BM) and normalizes TNF- $\alpha$ independently of methotrexate, and BVA is a source for obtaining the DPPIV enzyme [23]. BVA (Species: A. mellifera, AR: ST36 acupoint, dose: 
$0.8 \mathrm{mg} / \mathrm{kg}$, BV dissolved in a volume of $20 \mu \mathrm{L}$ saline) on spinal astrocyte activation is mediated by the activation of both $\alpha-2 \mathrm{~A}$ and $\alpha-2 \mathrm{C}$ adrenoceptors, which in turn results in a potent analgesic effect [24]. BVA (Species: A. mellifera, AR: ST36 acupoint, dose: $0.8 \mathrm{mg} / \mathrm{kg}$ ( $1 \mathrm{~K}$, diluted by saline with ratio of 1:1000)) in an inflammatory pain model could be significantly improved by modulation of adrenal medulla-derived epinephrine, and this effect is mediated by peripheral $\beta$-adrenoceptors [25]. BVA (Species: A. mellifera, AR: ST36 acupoint, dose: $1.0 \mathrm{mg} / \mathrm{kg}$ ) can relieve collagen type-II-induced pain via the partial involvement of the $\delta$-opioid and $\alpha 2$-adrenergic receptors [26].

(3) In cases of neuropathic pain, BVA (Species: A. mellifera, AR: GB39, LI4, LV3, and SJ5 acupoints, dose: $0.1 \mathrm{mg} / \mathrm{mL}$ BVA was injected, total volume of $9.6 \mathrm{~mL}$ in 6 sessions) may demonstrate functions via mechanisms similar to those observed with norepinephrine reuptake inhibitors or adrenergic reuptake inhibitors [30]. Combination treatment with BVA (Species: A. mellifera, AR: ST36 acupoint, dose: $1.0 \mathrm{mg} / \mathrm{kg}, \mathrm{BV}$ dissolved in a volume of $20 \mu \mathrm{L}$ PBS) and venlafaxine (AR: oral route, dose: $40 \mathrm{mg} / \mathrm{kg}$, venlafaxine dissolved in a saline) can help relieve paclitaxel-induced cold and mechanical allodynia through spinal 5-HT1/5-HT2, 5-HT3, and $\alpha 2$-adrenergic receptors [36]. The combined effect of BVA (Species: A. mellifera, $1.0 \mathrm{mg} / \mathrm{kg}$ was injected into ST36 acupoint in a volume of $20 \mu \mathrm{L}$ ) and morphine (AR: intraperitoneal route, dose: $2.0 \mathrm{mg} / \mathrm{kg}$ in a volume of $0.2 \mathrm{~mL}$ ) on oxaliplatin-induced cold and mechanical allodynia is mediated by a spinal opioidergic and 5-HT3 receptors [37]. BVA (Species: A. mellifera, AR: ST36 acupoint, dose: $1.0 \mathrm{mg} / \mathrm{kg}$, BV dissolved in PBS at concentrations of $1 \mathrm{mg} / \mathrm{mL}$ ) may attenuate oxaliplatin-induced neuropathic pain by altering the action potential threshold in A-fibre dorsal root ganglia neurons [43]. BVA (Species: A. mellifera, AR: ST36 acupoint, dose: $1.0 \mathrm{mg} / \mathrm{kg}$, BV dissolved in volume of $50 \mu \mathrm{L}$ saline)-induced analgesia is mediated by the descending noradrenergic pathway, which mainly originates from the locus coeruleus (LC) [46].

$\mathrm{PLA}_{2}$ (Species: A. mellifera, AR: intraperitoneal route, dose: $0.2 \mathrm{mg} / \mathrm{kg}, \mathrm{PLA}_{2}$ dissolved in a PBS) can help reduce spinal nerve injury-induced mechanical and cold allodynia through the activation of spinal $\alpha 1$-adrenergic receptors [33]. PLA 2 (Species: A. mellifera, AR: not reported, dose: $0.2 \mathrm{mg} / \mathrm{kg}, \mathrm{PLA}_{2}$ dissolved in a PBS) reduces oxaliplatin-induced mechanical and cold allodynia through activation of $\alpha 2$-adrenergic receptors [38]. PLA 2 (Species: A. mellifera, AR: intraperitoneal route, dose: $0.2 \mathrm{mg} / \mathrm{kg}$ ) may prevent oxaliplatininduced mechanical and cold allodynia by suppressing immune responses in the dorsal root ganglia (DRG) by tregs [39].

Melittin (Species: A. mellifera, AR: ST36 acupoint, dose: $0.5 \mathrm{mg} / \mathrm{kg}$, melittin dissolved in a saline) demonstrates an analgesic effect on oxaliplatin-induced peripheral neuropathy, and its effect is mediated by activation of the spinal $\alpha 1$ and $\alpha 2$-adrenergic receptors [42].

(4) In cases of mechanical allodynia, BVA (Species: A. mellifera, AR: ST36 acupoint, dose: $1.0 \mathrm{mg} / \mathrm{kg}, \mathrm{BV}$ dissolved in a PBS) demonstrates potent suppressive effects in paclitaxel-induced mechanical allodynia, which is mediated by the spinal $\alpha 2$-adrenergic receptor [35].

The anti-allodynic effect of BVA (Species: A. mellifera, AR: ST36 acupoint, dose: $0.1 \mathrm{mg} / \mathrm{kg}, \mathrm{BV}$ dissolved in a volume of $20 \mu \mathrm{L}$ saline) is related to the activation of peripheral nerves in a BVA-injected site, and it is also meditated by the activation of alpha-2 adrenoceptors and opioid receptors in the spinal cord [40]. Repetitive BVA (Species: $A$. mellifera, AR: ST36 acupoint, dose: $0.1 \mathrm{mg} / \mathrm{kg}$, BV dissolved in a volume of $20 \mu \mathrm{L}$ saline) injected 18 times gradually and significantly decreases oxaliplatin-induced mechanical allodynia and recovers the loss of intraepidermal nerve fibres (IENFs) in neuropathic mice through an $\alpha 2$-adrenoceptor mechanism [41]. The analgesic effects of repetitive BVA (Species: A. mellifera, AR: ST36 acupoint, dose: $0.25 \mathrm{mg} / \mathrm{kg}, \mathrm{BV}$ dissolved in a volume of $50 \mu \mathrm{L}$ saline) injected seven times are related to the activation of the LC noradrenergic system and a decrease in spinal phosphorylation [44]. The repetitive application of BVA (Species: A. mellifera, AR: ST36 acupoint, dose: $0.25 \mathrm{mg} / \mathrm{kg}$, BV dissolved in a volume of $50 \mu \mathrm{L}$ saline) suppressed SCI-induced mechanical allodynia, which is mediated by the suppression of spinal astrocytes or microglial activation [45]. 
(5) In cases of cold allodynia, BVA (Species: A. mellifera, AR: GV3 acupoint, dose: $0.25 \mathrm{mg} / \mathrm{kg}$, BV dissolved in a volume of 0.05 cc saline) relieves oxaliplatin-induced cold allodynia through activation of the serotonergic system, especially spinal 5-HT3 receptors [47]. Spinal $\alpha 4 \beta 2$ receptors mediate the suppressive effect of BVA (Species: A. mellifera, AR: GV3 acupoint, dose: $1.0 \mathrm{mg} / \mathrm{kg}$, BV dissolved in a volume of $0.05 \mathrm{cc}$ saline) on oxaliplatin-induced cold allodynia [48]. The anti-allodynic effect of BVA (Species: A. mellifera, AR: GV3 acupoint, dose: $1.0 \mathrm{mg} / \mathrm{kg}$, BV dissolved in a volume of $0.05 \mathrm{cc}$ saline) on oxaliplatin-induced cold allodynia is mediated by the noradrenergic system, and not the opioid system [49]. BVA (Species: A. mellifera, AR: ST36 acupoint, dose: $2.5 \mathrm{mg} / \mathrm{kg}$, BV dissolved in a volume of $50 \mu \mathrm{L}$ saline) could reduce cold allodynia through activation of spinal $\alpha 2$-adrenoceptors in chronic constrictive injury-induced rats [50].

(6) In cases of post-ischaemic pain, the anti-allodynic effect of BVA (Species: A. mellifera, AR: dorsum of the ipsilateral hind paw, dose: $1.0 \mathrm{mg} / \mathrm{kg} \mathrm{BV}$ injected 1, 2, and 3 times) has been objectively demonstrated through a decrease in the increased expression of neurokinin type 1 (NK-1) receptors in the DRG [53].

(7) In cases of prostatic pain, melittin (Species: A. mellifera, AR: both right and left lobes of the prostate, dose: $0.05 \mathrm{mg}$ melittin in a volume of $0.2 \mathrm{~mL}$ saline) alleviates CFA-induced prostatitis pain by suppressing cyclooxygenase-2 expression [53].

\section{Discussion}

In clinical settings, the existing literature suggests that BVA monotherapy or a combination with CT (e.g., NSAID and PT) can help improve musculoskeletal and neuropathic pain. Additionally, only minor adverse events such as pruritus, burning sensation, local swelling, and aching were observed in the BVA treatment groups, and fatal reactions such as anaphylaxis did not occur [56]. Anaphylaxis was not observed in clinical trials with relatively small sample sizes, because of a low incidence of $0.014 \%$ [57]. Patients who have a high risk of developing anaphylaxis or hypersensitivity were excluded according to the skin tests, and the history of patients was documented [35]. The safety of BVA is a crucial factor in deciding the applicability of BV as a therapeutic agent [11]. Therefore, when using BVA in humans, it is only recommended for use after determining the possibility of occurrence of allergic reactions by considering the conduction of a skin test and by documenting treatment history and family history.

The incidence of side effects of bee sting therapy (BST) was 7.5-98.83\%, with a large variation [58-60]. In case of BVA, the incidence of AEs was 0.03-18.18\% [61,62]. In order to use BV clinically, safety must be guaranteed. The BST is a treatment that stimulates the skin with live bee sting [35]. The BVA is the injection of purified and diluted BV from worker bees into the acupuncture points of human [35]. Thus, BVA corresponds to a treatment developed to produce a therapeutic effect while reducing side effects when clinically applying BV compared to BST. However, there may be deviations in the occurrence of side effects due to dilution concentration, injection dose, and sensitivity to BV. Further studies on prediction factors to prevent AEs of BVA will be investigated.

In most clinical studies, it was reported that BVA was diluted with saline at a certain ratio and injected to patients. In experimental studies, BVA was used diluted in saline or PBS, or used without dilution. It is possible that undiluted BVA was injected into mice or rats, or diluted BVA was injected but not mentioned in the article. Therefore, it is necessary to specify the dilution method and used concentration of BVA in future clinical or experimental studies. In addition, we look forward to future studies on any differences in effectiveness and safety depending on the concentration of BVA and the substances used for dilution.

Among the 40 studies included in this review, 39 were conducted using BV, $\mathrm{PLA}_{2}$, and melittin extracted from A. mellifera. Honeybees (A. mellifera) can be found worldwide, and there is considerable genetic diversity in global A. mellifera populations [63,64]. Additionally, depending on the environmental differences such as the region, climate, and flower distribution within the same country, differences in the composition and concentration of 
BV may be observed. As BV is a natural toxin, a certain level of standardisation is necessary for its use as a raw material for drug development and clinical and experimental studies.

The pain reduction effects and mechanisms of collagen-, adjuvant-, and formalininduced inflammatory pain in a mouse model [22-26] were confirmed through animal studies. However, all included studies confirmed the treatment effect of inflammatory pain following a single BVA treatment. In future studies, the number of administrations and treatment periods should be extended to identify conditions that can help produce the optimal treatment effect. In another study [24], high-concentration BVA $(0.8 \mathrm{mg} / \mathrm{kg})$ exerted a significant analgesic effect compared to low-concentration BVA $(0.08 \mathrm{mg} / \mathrm{kg})$, and in another study [26], low-concentration BVA $(1 \mathrm{mg} / \mathrm{kg})$ exerted a significant analgesic effect. It has been reported to be more effective than BVA ( $2 \mathrm{mg} / \mathrm{kg})$. The concentration of venom correlates to both therapeutic efficacy and adverse allergic and toxic reactions. We anticipate the conduction of further studies for examination of the effect of BV on the treatment of inflammatory pain, considering BV concentration.

Prior to 2010, pain-related clinical studies of BVA focused on osteoarthritis, rheumatoid arthritis, and musculoskeletal pain (e.g., low back, neck, and shoulder pain, acute ankle or wrist sprain, and pain of a herniated lumbar disc) [13]. In animal studies, most experimental studies have been conducted using inflammatory nociception model mice induced by collagen, adjuvant, and formalin, and only one study was conducted using neuropathic pain model mice induced by chronic constriction injury [13]. On the other hand, in the last 10 years, clinical studies have been conducted on various aspects and types of neuralgia, and experimental studies have been conducted on mechanical and cold allodynia. Studies have been conducted on the following: (1) post-stroke pain [27], chemotherapy-induced peripheral neuropathic pain $[29,30]$, complex regional pain syndrome [31], refractory postherpetic neuralgia [32], and intervertebral disc disease-induced neurological pain in clinical fields [51]; (2) mechanical allodynia induced by SNL [33,34], CCI [44-46], and chemotherapy drugs including oxaliplatin [37-43] and paclitaxel [35,36] in animal studies; (3) cold allodynia induced by SNL [34,35], CCI [50], and chemotherapy drugs including oxaliplatin [37-39,42,43,47-49], paclitaxel [36], and vincristine [46] in animal experimental studies. Multiple studies have been conducted on neuropathic pain induced by chemotherapy drugs. Approximately $66 \%$ of the cancer patients experience mild to severe pain during treatment $[65,66]$. This suggests that BVA could be utilized to treat chemotherapy-induced pain, and we anticipate the conduction of further research on various chemotherapy-induced pain conditions. Pain is one of the most common symptoms in humans, and pain, especially neuropathic pain and chemotherapy-induced pain, can affect daily life and economic activities [67]. It is not necessary to utilize only one method for the treatment of pain, nor is it necessary to change the conventional drugs to BVA. As BVA is not consumed orally, and is instead injected into acupoints with a syringe, it can be considered as a viable alternative intervention for patients presenting with pain who encounter problems with oral administration. The treatment effects and mechanisms of anti-nociception have been well characterised in articles published on the effects of BVA on pain. We look forward to using BVA as one of the treatments that can be used to treat pain; additionally, we aim to investigate various pain conditions in clinical, experimental analyses using BVA.

BV injection on acupoint ST36 has been reported in ten experimental studies [35-37,40$43,45,46,49]$. The ST36 is located on the leg, $3 \mathrm{~cm}$ below the knee, and its functions in Oriental medicine involve the fortification of the stomach and spleen, the replenishment of qi and nourishing blood, clearance and activation of meridians, and nourishment of the channels $[68,69]$. Although the target disease, treatment concentration, and dose of BVA are extremely important, the treatment effect may vary depending on the acupuncture point at which the treatment is performed from the perspective of Oriental medicine. Lim et al. [49] assessed the analgesic effects of oxaliplatin-induced cold allodynia by administering BV at three acupoints (GV3, LI11, and ST36), and found that the duration of treatment effect was statistically significant in the GV3 group compared to other groups. As BVA is a therapeutic 
strategy intended for injecting BV on acupuncture points, additional research considering this point is warranted.

\section{Conclusions}

In the past decade, numerous experimental and clinical studies have been reporting the therapeutic potential of BVA for musculoskeletal pain, inflammatory pain, neuropathic pain, post-ischaemic pain, and prostatic pain. This paper will be the basis for promoting research that will make BVA more general and safer. However, several experimental results have still not been investigated in humans. The effectiveness and safety of BVA for various types of pain have to be supported by clinical trials. In addition, in most cases, the optimal dose and frequency duration of BVA still need to be determined for optimum effectiveness and safety.

Author Contributions: Conceptualisation, S.-H.S. and G.L.; methodology, S.-H.S. and G.L.; writingoriginal draft, S.-H.S.; writing - review and editing, G.L.; visualisation, S.-H.S. and G.L.; supervision, G.L. All authors have read and agreed to the published version of the manuscript.

Funding: This work was supported by the National Research Foundation of Korea (NRF) grant funded by the Korea government (MSIT) (No. 2019R1G1A1100651) and by the Policy Support Project for the Development of Traditional Korean Medicine funded by the Ministry of Health and Welfare.

Institutional Review Board Statement: Not applicable.

Informed Consent Statement: Not applicable.

Data Availability Statement: The data will be made available upon reasonable request.

Acknowledgments: We would like to appreciate the editor and all staff working in the editorial office of Toxins. We also appreciate all anonymous reviewers who provided insightful suggestions for our manuscript.

Conflicts of Interest: The authors declare that there are no conflicts of interest regarding the publication of this paper.

\section{References}

1. Goldberg, D.S.; McGee, S.J. Pain as a global public health priority. BMC Public Health 2011, 11, 770. [CrossRef] [PubMed]

2. Jackson, T.; Thomas, S.; Stabile, V.; Han, X.; Shotwell, M.; McQueen, K. Prevalence of chronic pain in low-income and middleincome countries: A systematic review and meta-analysis. Lancet 2015, 385 (Suppl. 2), S10. [CrossRef]

3. Cho, S.-H.; Kim, Y.-M.; Lee, J.-H.; Kim, H.-S.; Song, J.-S. The trend of prevalence of pain in Korea from 2005 to 2016. Korean J. Pain 2020, 33, 352-358. [CrossRef]

4. Zelaya, C.; Dahlhamer, J.M.; Lucas, J.W.; Connor, E.M. Chronic Pain and High-Impact Chronic Pain Among U.S. Adults, 2019; NCHS Data Brief, no. 390; National Center for Health Statistics: Hyattsville, MD, USA, 2020.

5. Todd, A.; McNamara, C.L.; Balaj, M.; Huijts, T.; Akhter, N.; Thomson, K.; Kasim, A.; Eikemo, T.A.; Bambra, C. The European epidemic: Pain prevalence and socioeconomic inequalities in pain across 19 European countries. Eur. J. Pain 2019, 23, 1425-1436. [CrossRef] [PubMed]

6. Lipman, A.G. Pain as a human right: The 2004 Global Day Against Pain. J. Pain Palliat. Care Pharmacother. 2005, 19, 85-100. [CrossRef]

7. International Association for the Study of Pain. Available online: https://www.iasp-pain.org/PublicationsNews/NewsDetail. aspx?ItemNumber $=10475$ (accessed on 12 April 2021).

8. Lohman, D.; Schleifer, R.; Amon, J.J. Access to pain treatment as a human right. BMC Med. 2010, 8, 8. [CrossRef] [PubMed]

9. King, N.B.; Fraser, V. Untreated Pain, Narcotics Regulation, and Global Health Ideologies. PLoS Med. 2013, 10 , e1001411. [CrossRef]

10. Lee, H.J. Principle of Herbal Acupuncture and Clinical Application; Iljoongsa: Seoul, Korea, 1999.

11. Kim, H.J.; Park, S.Y.; Lee, G. Potential therapeutic applications of bee venom on skin disease and its mechanisms: A literature review. Toxins 2019, 11, 374. [CrossRef]

12. Jang, S.; Kim, K.H. Clinical Effectiveness and Adverse Events of Bee Venom Therapy: A Systematic Review of Randomized Controlled Trials. Toxins 2020, 12, 558. [CrossRef]

13. Chen, J.; Lariviere, W.R. The nociceptive and anti-nociceptive effects of bee venom injection and therapy: A double-edged sword. Prog. Neurobiol. 2010, 92, 151-183. [CrossRef]

14. Shen, L.; Lee, J.H.; Joo, J.C.; Park, S.J.; Song, Y.S. Bee Venom Acupuncture for Shoulder Pain: A Systematic Review and Meta-analysis of Randomized Controlled Trials. J. Pharmacopunct. 2020, 23, 44-53. 
15. An, K.; Kim, Y.S.; Kim, H.Y.; Lee, H.; Hahm, D.H.; Lee, K.S.; Kil Kang, S. Needle-free acupuncture benefits both patients and clinicians. Neurol. Res. 2010, 32 (Suppl. 1), 22-26. [CrossRef] [PubMed]

16. Koh, P.S.; Seo, B.K.; Cho, N.S.; Park, H.S.; Park, D.S.; Baek, Y.H. Clinical effectiveness of bee venom acupuncture and physiotherapy in the treatment of adhesive capsulitis: A randomized controlled trial. J. Shoulder Elb. Surg. 2013, 22, 1053-1062. [CrossRef] [PubMed]

17. Park, Y.C.; Koh, P.S.; Seo, B.K.; Lee, J.W.; Cho, N.S.; Park, H.S.; Park, D.S.; Baek, Y.H. Long-Term Effectiveness of Bee Venom Acupuncture and Physiotherapy in the Treatment of Adhesive Capsulitis: A One-Year Follow-Up Analysis of a Previous Randomized Controlled Trial. J. Altern. Complement. Med. 2014, 20, 919-924. [CrossRef]

18. Seo, B.K.; Han, K.; Kwon, O.; Jo, D.J.; Lee, J.H. Efficacy of bee venom acupuncture for chronic low back pain: A randomized, double-blinded, sham-controlled trial. Toxins 2017, 9, 361. [CrossRef] [PubMed]

19. Shin, B.-C.; Kong, J.C.; Park, T.-Y.; Yang, C.-Y.; Kang, K.-W.; Choi, S.-M. Bee venom acupuncture for chronic low back pain: A randomised, sham-controlled, triple-blind clinical trial. Eur. J. Integr. Med. 2012, 4, e271-e280. [CrossRef]

20. Shin, J.S.; Lee, J.; Kim, M.R.; Jung, J.; Shin, B.C.; Lee, M.S.; Ha, I.H. The short-term effect of integrated complementary and alternative medicine treatment in inpatients diagnosed with lumbar intervertebral disc herniation: A prospective observational study. J. Altern. Complement. Med. 2016, 22, 533-543. [CrossRef]

21. Conrad, V.J.; Hazan, L.L.; Latorre, A.J.; Jakubowska, A.; Kim, C.M. Efficacy and Safety of Honey Bee Venom (Apis mellifera) Dermal Injections to Treat Osteoarthritis Knee Pain and Physical Disability: A Randomized Controlled Trial. J. Altern. Complement. Med. 2019, 25, 845-855. [CrossRef]

22. Nipate, S.S.; Hurali, P.B.; Ghaisas, M.M. Evaluation of anti-inflammatory, anti-nociceptive, and anti-arthritic activities of Indian Apis dorsata bee venom in experimental animals: Biochemical, histological, and radiological assessment. Immunopharmacol. Immunotoxicol. 2015, 37, 171-184. [CrossRef]

23. Yamasaki, S.C.; Mendes, M.T.; Alponti, R.F.; Silveira, P.F. Efficacy of parenteral administration of bee venom in experi-mental arthritis in the rat: A comparison with methotrexate. Toxicon 2015, 98, 75-88. [CrossRef] [PubMed]

24. Kang, S.Y.; Kim, C.Y.; Roh, D.H.; Yoon, S.Y.; Park, J.H.; Lee, H.J.; Beitz, A.J.; Lee, J.H. Chemical stimulation of the ST36 acupoint reduces both formalin-induced nociceptive behaviors and spinal astrocyte activation via spinal alpha-2 adrenoceptors. Brain Res Bull. 2011, 86, 412-421. [CrossRef] [PubMed]

25. Kang, S.Y.; Roh, D.H.; Kim, H.W.; Han, H.J.; Beitz, A.J.; Lee, J.H. Blockade of adrenal medulla-derived epinephrine po-tentiates bee venom-induced antinociception in the mouse formalin test: Involvement of peripheral $\beta$-adrenoceptors. Evid.-Based Complement. Altern. Med. 2013, 2013, 809062. [CrossRef] [PubMed]

26. Huh, J.-E.; Seo, B.-K.; Lee, J.-W.; Park, Y.-C.; Baek, Y.-H. Analgesic Effects of Diluted Bee Venom Acupuncture Mediated by $\delta$-Opioid and $\alpha 2$-Adrenergic Receptors in Osteoarthritic Rats. Altern. Ther. Health Med. 2018, 24, 28-35. [PubMed]

27. Lim, S.M.; Lee, S.-H. Effectiveness of bee venom acupuncture in alleviating post-stroke shoulder pain: A systematic review and meta-analysis. J. Integr. Med. 2015, 13, 241-247. [CrossRef]

28. Cho, S.-Y.; Park, J.-Y.; Jung, W.-S.; Moon, S.-K.; Park, J.-M.; Ko, C.-N.; Park, S.-U. Bee venom acupuncture point injection for central post stroke pain: A preliminary single-blind randomized controlled trial. Complement. Ther. Med. 2013, 21, 155-157. [CrossRef]

29. Park, J.W.; Jeon, J.H.; Yoon, J.; Jung, T.Y.; Kwon, K.R.; Cho, C.K.; Lee, Y.W.; Sagar, S.; Wong, R.; Yoo, H.S. Effects of sweet bee venom pharmacopuncture treatment for chemotherapy-induced peripheral neuropathy: A case series. Integr. Cancer Ther. 2012, 11, 166-171. [CrossRef]

30. Yoon, J.; Jeon, J.H.; Lee, Y.W.; Cho, C.K.; Kwon, K.R.; Shin, J.E.; Sagar, S.; Wong, R.; Yoo, H.S. Sweet bee venom phar-macopuncture for chemotherapy-induced peripheral neuropathy. J. Acupunct. Meridian. Stud. 2012, 5, 156-165. [CrossRef]

31. Kim, J.-M.; Jeon, H.-J.; Kim, H.-J.; Cho, C.-K.; Yoo, H.-S. Bee Venom Pharmacopuncture: An Effective Treatment for Complex Regional Pain Syndrome. J Pharmacopunct. 2014, 17, 66-69. [CrossRef] [PubMed]

32. Lee, S.M.; Lim, J.; Lee, J.D.; Choi, D.Y.; Lee, S. Bee venom treatment for refractory postherpetic neuralgia: A case report. J. Altern. Complement. Med. 2014, 20, 212-214. [CrossRef] [PubMed]

33. Woo, S.; Chung, G.; Bae, H.; Kim, S.K. Suppressive effects of bee venom-derived phospholipase A2 on mechanical allo-dynia in a rat model of neuropathic pain. Toxins 2019, 11, 477. [CrossRef]

34. Koh, W.U.; Choi, S.S.; Lee, J.H.; Lee, S.H.; Lee, S.K.; Lee, Y.K.; Leem, J.G.; Song, J.G.; Shin, J.W. Perineural pretreatment of bee venom attenuated the development of allodynia in the spinal nerve ligation injured neuropathic pain model; an experimental study. BMC Complement. Altern. Med. 2014, 14, 431. [CrossRef] [PubMed]

35. Choi, J.; Jeon, C.; Lee, J.H.; Jang, J.U.; Quan, F.S.; Lee, K.; Kim, W.; Kim, S.K. Suppressive effects of bee venom acupuncture on paclitaxel-induced neuropathic pain in rats: Mediation by spinal $\alpha_{2}$-adrenergic receptor. Toxins 2017, 9, 351. [CrossRef] [PubMed]

36. Li, D.; Yoo, J.H.; Kim, S.K. Long-Lasting and Additive Analgesic Effects of Combined Treatment of Bee Venom Acupuncture and Venlafaxine on Paclitaxel-Induced Allodynia in Mice. Toxins 2020, 12, 620. [CrossRef] [PubMed]

37. Kim, W.; Kim, M.J.; Go, D.; Min, B.-I.; Na, H.S.; Kim, S.K. Combined Effects of Bee Venom Acupuncture and Morphine on Oxaliplatin-Induced Neuropathic Pain in Mice. Toxins 2016, 8, 33. [CrossRef]

38. Li, D.; Lee, Y.; Kim, W.; Lee, K.; Bae, H.; Kim, S.K. Analgesic Effects of Bee Venom Derived Phospholipase A2 in a Mouse Model of Oxaliplatin-Induced Neuropathic Pain. Toxins 2015, 7, 2422-2434. [CrossRef]

39. Li, D.; Kim, W.; Shin, D.; Jung, Y.; Bae, H.; Kim, S.K. Preventive effects of bee venom derived phospholipase $A_{2}$ on ox-aliplatininduced neuropathic pain in mice. Toxins 2016, 8, 27. [CrossRef] 
40. Yoon, S.-Y.; Yeo, J.-H.; Han, S.-D.; Bong, D.-J.; Oh, B.; Roh, D.-H. Diluted Bee Venom Injection Reduces Ipsilateral Mechanical Allodynia in Oxaliplatin-Induced Neuropathic Mice. Biol. Pharm. Bull. 2013, 36, 1787-1793. [CrossRef] [PubMed]

41. Yeo, J.-H.; Yoon, S.-Y.; Kwon, S.-K.; Kim, S.-J.; Lee, J.-H.; Beitz, A.; Roh, D.-H. Repetitive Acupuncture Point Treatment with Diluted Bee Venom Relieves Mechanical Allodynia and Restores Intraepidermal Nerve Fiber Loss in Oxaliplatin-Induced Neuropathic Mice. J. Pain 2015, 17, 298-309. [CrossRef] [PubMed]

42. Choi, S.; Chae, H.K.; Heo, H.; Hahm, D.H.; Kim, W.; Kim, S.K. Analgesic effect of melittin on oxaliplatin-induced peripheral neuropathy in rats. Toxins 2019, 11, 396. [CrossRef] [PubMed]

43. Lee, J.H.; Gang, J.; Yang, E.; Kim, W.; Jin, Y.-H. Bee Venom Acupuncture Attenuates Oxaliplatin-Induced Neuropathic Pain by Modulating Action Potential Threshold in A-Fiber Dorsal Root Ganglia Neurons. Toxins 2020, 12, 737. [CrossRef]

44. Kang, S.-Y.; Roh, D.-H.; Yoon, S.-Y.; Moon, J.-Y.; Kim, H.-W.; Lee, H.-J.; Beitz, A.J.; Lee, J.-H. Repetitive Treatment with Diluted Bee Venom Reduces Neuropathic Pain Via Potentiation of Locus Coeruleus Noradrenergic Neuronal Activity and Modulation of Spinal NR1 Phosphorylation in Rats. J. Pain 2012, 13, 155-166. [CrossRef]

45. Kang, S.-Y.; Roh, D.-H.; Choi, J.-W.; Ryu, Y.; Lee, J.-H. Repetitive Treatment with Diluted Bee Venom Attenuates the Induction of Below-Level Neuropathic Pain Behaviors in a Rat Spinal Cord Injury Model. Toxins 2015, 7, 2571-2585. [CrossRef] [PubMed]

46. Li, D.; Chung, G.; Kim, S.K. The involvement of central noradrenergic pathway in the analgesic effect of bee venom acupuncture on vincristine-induced peripheral neuropathy in rats. Toxins 2020, 12, 775. [CrossRef] [PubMed]

47. Lee, J.-H.; Li, D.X.; Yoon, H.; Go, D.; Quan, F.S.; Min, B.-I.; Kim, S.K. Serotonergic mechanism of the relieving effect of bee venom acupuncture on oxaliplatin-induced neuropathic cold allodynia in rats. BMC Complement. Altern. Med. 2014, 14, 471. [CrossRef] [PubMed]

48. Yoon, H.; Kim, M.J.; Yoon, I.; Li, D.X.; Bae, H.; Kim, S.K. Nicotinic Acetylcholine Receptors Mediate the Suppressive Effect of an Injection of Diluted Bee Venom into the GV3 Acupoint on Oxaliplatin-Induced Neuropathic Cold Allodynia in Rats. Biol. Pharm. Bull. 2015, 38, 710-714. [CrossRef] [PubMed]

49. Lim, B.-S.; Moon, H.J.; Li, D.X.; Gil, M.; Min, J.K.; Lee, G.; Bae, H.; Kim, S.K.; Min, B.-I. Effect of Bee Venom Acupuncture on Oxaliplatin-Induced Cold Allodynia in Rats. Evid.-Based Complement. Altern. Med. 2013, 2013, 1-8. [CrossRef]

50. Kang, S.Y.; Roh, D.H.; Park, J.H.; Lee, H.J.; Lee, J.H. Activation of spinal $\alpha$-adrenoceptors using diluted bee venom stimulation reduces cold allodynia in neuropathic pain rats. Evid.-Based Complement. Alternat. Med. 2012, 2012, 784713. [CrossRef]

51. Tsai, L.-C.; Lin, Y.-W.; Hsieh, C.-L. Effects of Bee Venom Injections at Acupoints on Neurologic Dysfunction Induced by Thoracolumbar Intervertebral Disc Disorders in Canines: A Randomized, Controlled Prospective Study. BioMed Res. Int. 2015, 2015, 363801. [CrossRef]

52. Lee, S.H.; Lee, J.M.; Kim, Y.H.; Choi, J.H.; Jeon, S.H.; Kim, D.K.; Jeong, H.D.; Lee, Y.J.; Park, H.J. Antiallodynic Effects of Bee Venom in an Animal Model of Complex Regional Pain Syndrome Type 1 (CRPS-I). Toxins 2017, 9, 285. [CrossRef] [PubMed]

53. Lin, L.; Zhu, B.-P.; Cai, L. Therapeutic effect of melittin on a rat model of chronic prostatitis induced by Complete Freund's Adjuvant. Biomed. Pharmacother. 2017, 90, 921-927. [CrossRef]

54. Korean Pharmacopuncture Institute. Pharmacopuncturology; Elsevier: Seoul, Korea, 2011.

55. Lee, Y.; Kim, S.-G.; Kim, I.-S.; Lee, H.-D. Standardization of the Manufacturing Process of Bee Venom Pharmacopuncture Containing Melittin as the Active Ingredient. Evid.-Based Complement. Altern. Med. 2018, 2018, 2353280. [CrossRef]

56. Park, J.H.; Yim, B.K.; Lee, J.H.; Lee, S.; Kim, T.H. Risk associated with bee venom therapy: A systematic review and me-ta-analysis. PLoS ONE 2015, 10, e0126971. [CrossRef]

57. Yang, M.-S.; Lee, S.-H.; Kim, T.-W.; Kwon, J.-W.; Lee, S.M.; Kim, S.-H.; Kwon, H.-S.; Park, C.-H.; Park, H.-W.; Kim, S.-S.; et al. Epidemiologic and clinical features of anaphylaxis in Korea. Ann. Allergy Asthma Immunol. 2008, 100, 31-36. [CrossRef]

58. Zhou, R.Y.; Tan, N.; Huang, S.G. Parallel kidney tonic decoction and bee venom therapy for ankylosing spondylitis in 40 cases. Guid. J. Tradit. Chin. Med. Pharm. 2009, 15, 40-41.

59. Yu, X.J. Cases of parallel bee acupuncture and bee products treatment for rare disease. Apic. China 2006, 57, 27-28.

60. Xiao, X.; Zhao, B.; Li, W. Analysis on the pattern of edema in initial patients treated by apitherapy. Apic. China 2013, 64, 53-55.

61. Choi, S.W.; Choi, S.U.; Oh, S.J. A clinical report of localized itching after treatment with sweet bee venom. J. Pharmacopunct. 2010, 13, 103-108. [CrossRef]

62. Hwang, Y.; Lee, B. Clinical study of anaphylaxis on bee venom acupuncture. J. Korean Acupunct. Moxibustion Soc. 2000, 17, 149-159.

63. Muli, E.; Patch, H.; Frazier, M.; Frazier, J.; Torto, B.; Baumgarten, T.; Kilonzo, J.; Kimani, J.N.; Mumoki, F.; Masiga, D.; et al. Evaluation of the Distribution and Impacts of Parasites, Pathogens, and Pesticides on Honey Bee (Apis mellifera) Populations in East Africa. PLoS ONE 2014, 9, e94459. [CrossRef]

64. Pucca, M.B.; Cerni, F.A.; Oliveira, I.S.; Jenkins, T.P.; Argemí, L.; Sørensen, C.V.; Ahmadi, S.; Barbosa, J.E.; Laustsen, A.H. Bee Updated: Current Knowledge on Bee Venom and Bee Envenoming Therapy. Front. Immunol. 2019, 10, 2090. [CrossRef]

65. Everdingen, M.H.V.D.B.-V.; Hochstenbach, L.M.; Joosten, E.A.; Tjan-Heijnen, V.C.; Janssen, D.J. Update on Prevalence of Pain in Patients with Cancer: Systematic Review and Meta-Analysis. J. Pain Symptom Manag. 2016, 51, 1070-1090. [CrossRef] [PubMed]

66. Caraceni, A.; Shkodra, M. Cancer Pain Assessment and Classification. Cancers 2019, 11, 510. [CrossRef] [PubMed]

67. McCarberg, B.H.; Nicholson, B.D.; Todd, K.H.; Palmer, T.; Penles, L. The Impact of Pain on Quality of Life and the Unmet Needs of Pain Management: Results from Pain Sufferers and Physicians Participating in an Internet Survey. Am. J. Ther. 2008, 15, 312-320. [CrossRef] [PubMed] 
68. World Health Organization. Standard Acupuncture Nomenclature, 2nd ed.; World Health Organization: Geneva, Switzerland, 1993.

69. Archer, T.; Jonsson, G.; Minor, B.G.; Post, C. Noradrenergic-serotonergic interactions and nociception in the rat. Eur. J. Pharmacol. 1986, 120, 295-307. [CrossRef] 